\title{
Whistler-Langmuir oscillitons and their relation to auroral hiss
}

\author{
K. Sauer and R. D. Sydora \\ Department of Physics, University of Alberta, Edmonton, T6G 2G7, Canada \\ Received: 2 March 2011 - Revised: 20 September 2011 - Accepted: 20 September 2011 - Published: 5 October 2011
}

\begin{abstract}
A new type of oscilliton (soliton with superimposed spatial oscillations) is described which arises in plasmas if the electron cyclotron frequency $\Omega_{\mathrm{e}}$ is larger than the electron plasma frequency $\omega_{\mathrm{e}}$, which is a typical situation for auroral regions in planetary magnetospheres. Both highfrequency modes of concern, the Langmuir and the whistler wave, are completely decoupled if they propagate parallel to the magnetic field. However, for oblique propagation two mixed modes are created with longitudinal and transverse electric field components. The lower mode (in the literature commonly called the whistler mode, e.g. Gurnett et al., 1983) has whistler wave characteristics at small wave numbers and asymptotically transforms into the Langmuir mode. As a consequence of the coupling between these two modes, with different phase velocity dependence, a maximum in phase velocity appears at finite wave number. The occurrence of such a particular point where phase and group velocity coincide creates the condition for the existence of a new type of oscillating nonlinear stationary structure, which we call the whistler-Langmuir (WL) oscilliton. After determining, by means of stationary dispersion theory, the parameter regime in which WL oscillitons exist, their spatial profiles are calculated within the framework of cold (non-relativistic) fluid theory. Particle-in-cell (PIC) simulations are used to demonstrate the formation of WL oscillitons which seem to play an important role in understanding electron beam-excited plasma radiation that is observed as auroral hiss in planetary magnetospheres far away from the source region.
\end{abstract}

Keywords. Magnetospheric physics (Plasma waves and instabilities)

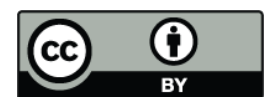

Correspondence to: K. Sauer

(ksauer@ualberta.ca)

\section{Introduction}

Oscillitons are soliton-like nonlinear structures superimposed by spatial oscillations. Since their first description in multi-ion plasmas (Sauer et al., 2001, 2003; Dubinin et al., 2002) and for the whistler wave branch (Sauer et al., 2002; Dubinin et al., 2003), there is a continuous effort to find out whether they are of similar physical relevance as the classical solitons in nonlinear media. Of particular interest is the question regarding their role in explaining the origin of waves measured in space. One obvious indication that oscillitons may be directly related to the generation process of plasma waves is the observation of wave packets in different frequency regimes. Up to now, the most compelling measurements have been done using the whistler wave branch. In the paper by Dubinin et al. (2007) the appearance of wave packets in the Earth's magnetosphere has been explained in terms of whistler oscillitons driven by temperature anisotropy. Subsequent kinetic simulations by Sydora et al. (2007) have shown that during the nonlinear saturation of the temperature anisotropy instability a significant wave number shift (from large $k$ values) to the Gendrin point (where phase and group velocity coincide) takes place accompanied by the formation of whistler oscillitons. As discussed in a recent paper by Sauer and Sydora (2010), a similar process happens during beam excitation of obliquely propagating whistler modes. The importance of whistler Gendrin modes has also been pointed out recently by Verkhoglyadova and Tsurutani (2009) showing that the measured propagation direction of a chorus event in the Earth's magnetosphere agrees well with the calculated Gendrin angle. In this respect it is remarkable that Gendrin mode radiation in the same whistler wave branch has been verified experimentally by Stenzel et al. (2008) in a large laboratory plasma. Oscilliton-like whistler wave packets below the electron cyclotron frequency occurred in an electron magnetohydrodynamics (EMHD) spheromak as a consequence of localized instabilities triggered by anisotropies of the electron distribution function.

Published by Copernicus Publications on behalf of the European Geosciences Union. 
In very recent papers the existence of oscillitons in wave modes other than whistlers has been studied. Ma and Hirose (2010) have shown that lower-hybrid (LH) oscillitons may evolve from ion-acoustic/ion-cyclotron solitary waves if in the underlying set of self-similar equations the effect of electron inertia is taken into account. Furthermore, the relevance of the LH solitary structures in explaining small-scale density depletions observed by rocket and satellite experiments has been discussed. Finally, Ma et al. (2011) have investigated the charge non-neutrality effects on the excitation of electrostatic ion-acoustic oscillitons.

With respect to Gendrin modes and their physical relevance, plasmas in the regime where the electron plasma frequency $\left(\omega_{\mathrm{e}}\right)$ is larger than the electron cyclotron frequency $\left(\Omega_{\mathrm{e}}\right)$, i.e. $G=\Omega_{\mathrm{e}} / \omega_{\mathrm{e}}<1$ have thus far been considered. However, for the auroral region of planetary magnetospheres the parameter regime is mainly such that $\Omega_{\mathrm{e}} \geq \omega_{\mathrm{e}}$ or $G \geq 1$. In order to include the large amount of satellite measurements made in this regime we have extended the analysis of stationary waves, in search of oscillitons, to high-frequency waves in plasmas with $G \geq 1$. With respect to wave phenomena known as auroral hiss (e.g. Gurnett, 1966; Gurnett et al., 1983), our main focus will be directed towards the frequency range around the electron plasma frequency. For parallel propagation three wave modes exist and they are the Langmuir, whistler and L-mode.

At oblique propagation the intersection of the whistler and Langmuir mode leads to mode splitting and associated formation of two mixed modes (commonly called the whistler and the Z-mode in the literature). Due to the merging of both modes with different phase velocity versus wave number dependence, the lower mode (henceforward called the whistlerLangmuir mode) gets a phase velocity maximum at finite wave number. This is a necessary condition for the existence of oscillating spatially growing structures, whose nonlinear identity is called the whistler-Langmuir oscilliton. Using linear dispersion theory we establish the parameter range in which they exist. The Maxwell-fluid approach is used to calculate the oscilliton profiles. In order to verify the existence of whistler-Langmuir (WL) oscillitons, particle-in-cell (PIC) simulations have been carried out using electron beams as a driver of the mainly electrostatic Langmuir waves. The results show that the onset of nonlinear saturation is accompanied by the excitation of waves whose wave number shifts more and more to the point of maximum phase velocity (generalized Gendrin point) where an accumulation of wave energy takes place. This behaviour illustrates the formation of WL oscillitons in the quasi-stationary state and represents the final nonlinear configuration of the beam-plasma interaction process.

The paper is organized as follows: in Sect. 2, the dispersion of high-frequency waves in plasmas with $\Omega_{\mathrm{e}}>\omega_{\mathrm{e}}$ is considered, concentrating mainly on whistler and Langmuir waves and their coupling at oblique propagation. In the view of later nonlinear theory, stationary waves are of special in- terest with emphasis on finding parameter regions that favour the existence of growing, spatially oscillating solutions. In Sect. 3 whistler-Langmuir (WL) oscillitons are described. These are nonlinear, stationary structures which arise due to the coupling between both underlying wave modes. Since whistler-Langmuir waves can be driven by electron beams, fluid dispersion theory is applied in Sect. 4 to study this mechanism of instability. As suggested by other whistler wave studies (Sydora et al., 2007; Sauer and Sydora, 2010), results of particle-in cell (PIC) simulations are presented in Sect. 5 which show that WL oscillitons are formed in the quasi-stationary saturated state of the beam-plasma interaction. Finally, we propose that WL oscillitons are a possible source of auroral hiss and is an alternative explanation to the commonly held view that auroral hiss originates from resonance cone conditions. This crucial point is discussed in the final Sect. 6.

\section{High-frequency waves in plasmas with $\Omega_{\mathrm{e}}>\omega_{\mathrm{e}}$}

\subsection{Dispersion relation}

The dispersion relation for high-frequency waves in a cold plasma in which the electron cyclotron frequency is larger than the electron plasma frequency $\left(\Omega_{\mathrm{e}}>\omega_{\mathrm{e}}\right)$ is derived using the same tensor formalism as described in the recent paper by Sauer and Sydora (2010). The only difference is that the displacement current has to be taken into account and therefore, the ratio between electron cyclotron frequency and electron plasma frequency $\left(G=\Omega_{\mathrm{e}} / \omega_{\mathrm{e}}\right)$ appears as an additional parameter.

Starting from the equation of motion of cold electrons (protons are considered as immobile background) and the Maxwell equations (see e.g. Baumjohann and Treumann, 1996), after linearization, one obtains

$$
\begin{aligned}
& \frac{\partial \boldsymbol{v}_{\mathrm{e}}}{\partial t}=-\frac{e}{m}\left(\boldsymbol{E}+\boldsymbol{v}_{\mathrm{e}} x \boldsymbol{B}_{0}\right) \\
& \frac{\partial \boldsymbol{B}}{\partial t}+\nabla x \boldsymbol{E}=0 \\
& -\frac{1}{c^{2}} \frac{\partial \boldsymbol{E}}{\partial t}+\nabla x \boldsymbol{B}=-\mu_{0} e n_{\mathrm{eo}} \boldsymbol{v}_{\mathrm{e}}
\end{aligned}
$$

Using the ansatz of periodic solutions $\boldsymbol{E}=$ $\boldsymbol{E}^{*}(\omega, k) \exp (-i \omega t+i \boldsymbol{k} \cdot \boldsymbol{x})$ one gets the following two vector equations for the electron velocity $\boldsymbol{V}_{\mathrm{e}}$ and the electric field $\mathbf{E}$ which are functions of $\omega$ (frequency) and $k$ (wave number)

$$
\begin{aligned}
& \boldsymbol{L}_{\mathrm{e}} \cdot \boldsymbol{V}_{\mathrm{e}}=c_{\mathrm{e}} \boldsymbol{E} \\
& \boldsymbol{F} \cdot \boldsymbol{E}=c_{j}\left(-N_{\mathrm{e}} \boldsymbol{V}_{\mathrm{e}}\right)
\end{aligned}
$$




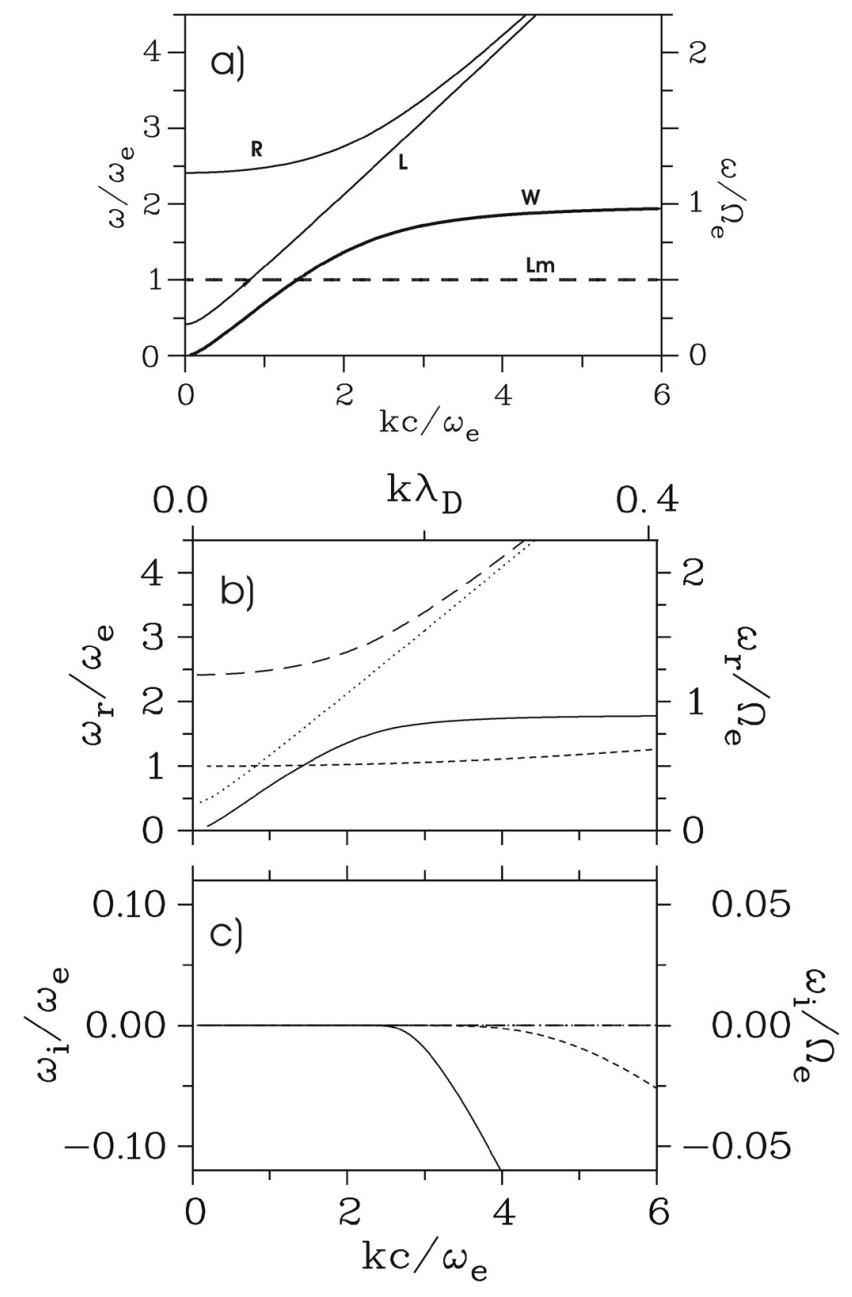

Fig. 1. Dispersion branches for parallel propagating waves, $G=2$. (a) Cold plasma theory, the four modes are the Langmuir mode (Lm), the whistler mode (W), the L-mode (L) and the R-mode upper branch $(\mathrm{R})$; (b) real and (c) imaginary part of frequency versus wave number from Vlasov approach using $\beta_{\mathrm{e}}=0.002$. The real frequencies of both approaches agree very well. The onset of kinetic damping of the whistler mode (solid line) for $k c / \omega_{\mathrm{e}}>1$ and the Langmuir mode (dashed line) in regions $k \lambda_{D} \geq 0.2\left(\lambda_{D}\right.$ is the Debye length) is clearly seen.

$-N_{\mathrm{e}} V_{\mathrm{e}}$ is the electron current in normalized units, $N_{\mathrm{e}}=1$. The other quantities, such as the matrices $\mathbf{L}_{e}, \mathbf{F}$ and the coefficients $c_{\mathrm{e}}, c_{j}$, are defined as follows:

$\mathbf{L}_{\mathrm{e}}=\left\{\left\{y^{2},-i y, 0\right\},\left\{i y, y^{2}, 0\right\},\left\{0,0, y^{2}\right\}\right\}$,

$\mathbf{F}=\left\{\left\{x^{2} \cos ^{2} \theta, 0,-x^{2} \sin \theta \cos \theta\right\},\left\{0, x^{2}, 0\right\}\right.$,

$$
\left.\left\{-x^{2} \sin \theta \cos \theta, 0, x^{2} \sin ^{2} \theta\right\}\right\}-G^{2} y^{2} \mathbf{I}
$$

where $c_{\mathrm{e}}=c_{j}=i y$ and $\mathbf{I}$ is the unit tensor: $\mathbf{I}=\{\{1,0,0\},\{0,1,0\},\{0,0,1\}\}$.

The undisturbed magnetic field $\boldsymbol{B}_{0}$ is in the z-direction and the propagation vector $\boldsymbol{k}=(k \sin \theta, 0, k \cos \theta)$ is inclined to it
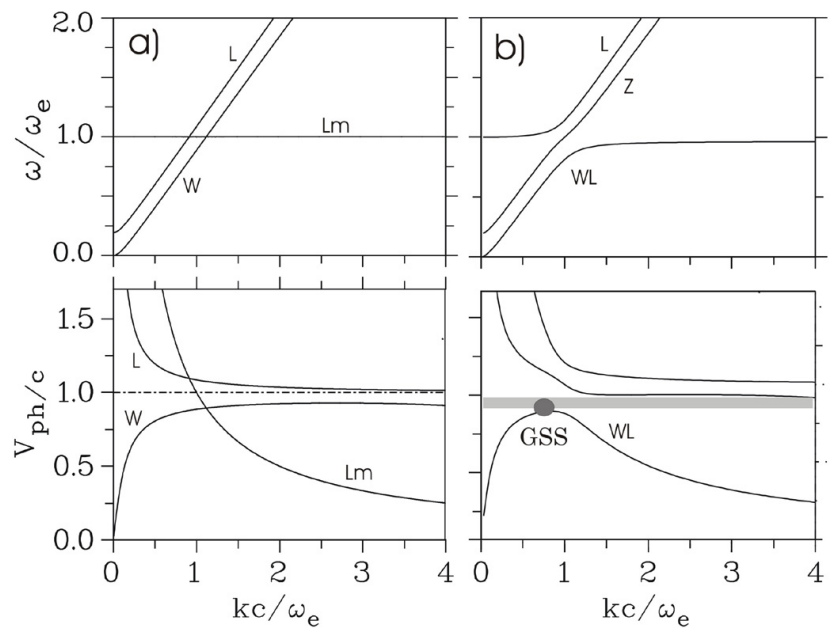

Fig. 2. Frequency $\omega / \omega_{\mathrm{e}}$ and phase velocity $V_{\mathrm{ph}} / c$ versus wave number $k c / \omega_{\mathrm{e}}$ for two propagation angles; (a) $\theta=0^{\circ}$, (b) $\theta=15^{\circ}$, $G=5$. Mode splitting which occurs at oblique propagation is clearly seen. Coupling between the whistler mode (W) and the Langmuir mode (Lm) leads to the whistler-Langmuir mode (WL). The original whistler mode at large wave numbers is named the Zmode. The GSS point in Fig. $2 \mathrm{~b}$ indicates maximum phase velocity. The gap above this point, drawn as a shaded grey strip, marks the region in which WL oscillitons are expected.

by the angle $\theta$. All quantities are written in normalized units; the frequency is in units of the electron cyclotron frequency, $y=\omega / \Omega_{\mathrm{e}}$, the wave number is normalized to the reciprocal electron skin length, $x=k c / \omega_{\mathrm{e}}$, the electron velocity is in units of the electron Alfvén velocity $V_{\mathrm{Ae}}=B_{0} /\left(\mu_{0} n_{\mathrm{e}} m_{\mathrm{e}}\right)^{1 / 2}$ and the electric field in units of $E_{0}=V_{\mathrm{Ae}} B_{0}$. By matrix multiplication of Eq. (2) with $\mathbf{L}_{\mathrm{e}}$ the electron velocity can be eliminated which results in the equation for $\boldsymbol{E}$ as $\mathbf{M} \cdot \mathbf{E}=0$ where the dispersion matrix $\mathbf{M}$ is given by $\mathbf{M}=\mathbf{L}_{e} \cdot \mathbf{F}-y^{2} \mathbf{I}$. The condition for a non-trivial solution of the electric field equation leads to the dispersion relation $D(y, x)=\operatorname{Det}[\mathbf{M}]=$ 0 . Finally, one gets a polynomial in $y$ (frequency) of fourth order in $z=y^{2}$ which can be written as

$D(y, x)=C_{0}+C_{1} z+C_{2} z^{2}+C_{3} z^{3}+C_{4} z^{4}$

with the coefficients

$$
\begin{aligned}
& C_{0}=-\cos ^{2} \theta x^{4}, \\
& C_{1}=1+\left(2+G^{2}\left(1+\cos ^{2} \theta\right)\right) x^{2}+\left(G^{2}+1\right) x^{4}, \\
& C_{2}=-3 G^{2}-G^{4}-\left(4 G^{2}+2 G^{4}\right) x^{2}-G^{2} x^{4}, \\
& C_{3}=3 G^{4}+G^{6}+2 G^{4} x^{2} \\
& C_{4}=-G^{6}
\end{aligned}
$$

The procedure is equivalent to the magnetoionic theory in which the dispersion relation is normally written in the form (e.g. Stix, 1992; Baumjohann and Treumann, 1996; Willes and Cairns, 2000)

$$
A N^{4}+B N^{2}+C=0
$$



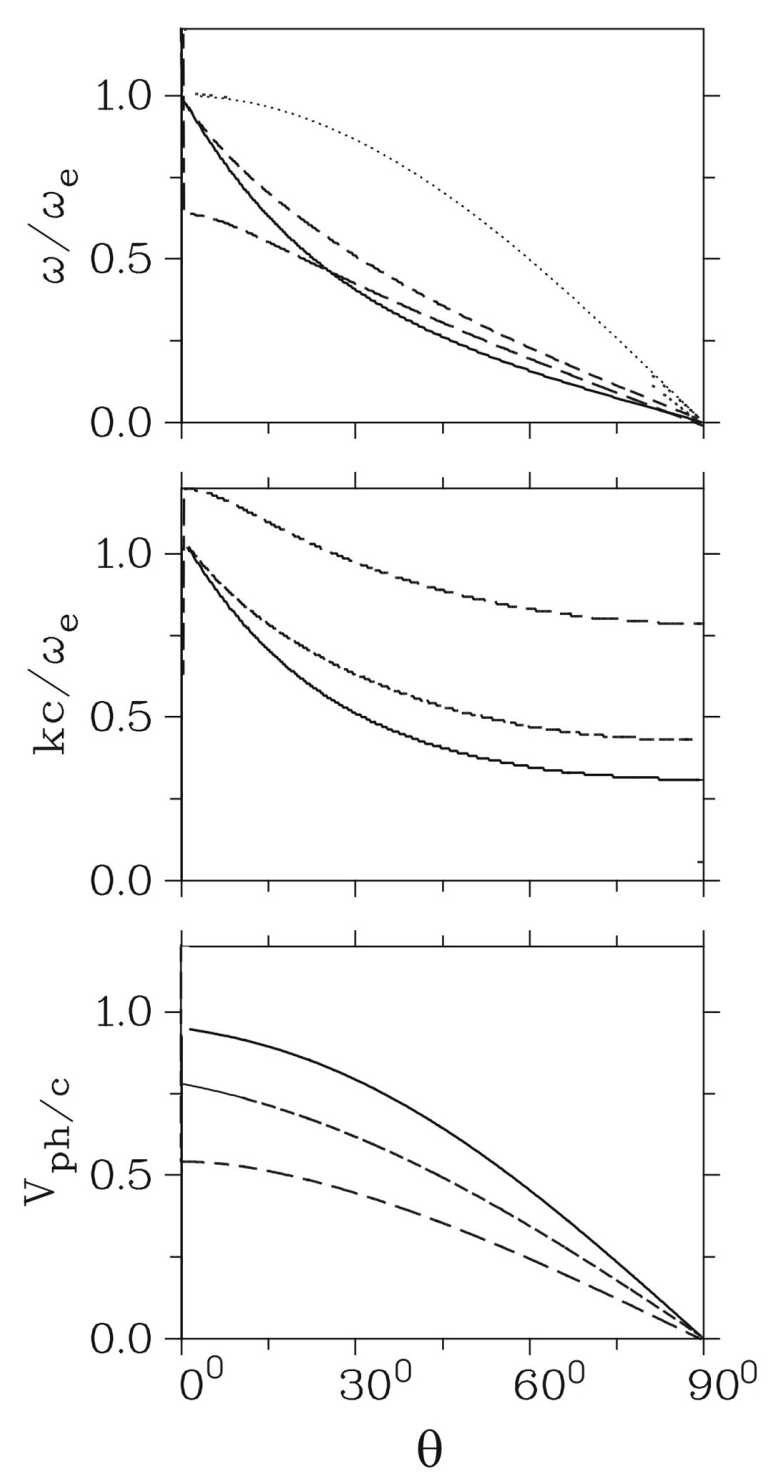

Fig. 3. Frequency, wave number and (maximum) phase velocity at the Gendrin point versus the propagation angle $\theta$ for three values of $G ; G=10$ (solid line), $G=2.5$ (short-dashed line) and $G=1.3$ (long-dashed line). The dotted curve in the upper panel represents the frequency dependence according to the resonance cone formula.

$(N=k c / \omega)$ with $A=S \sin ^{2} \theta+P \cos ^{2} \theta, \quad B=\left(S^{2}-\right.$ $\left.D^{2}\right) \sin ^{2} \theta+P S\left(1+\cos ^{2} \theta\right)$ and $C=P\left(S^{2}-D^{2}\right)$ where $S$, $D$ and $P$ are given by $S=(1 / 2)(R+L), D=(1 / 2)(R-$ $L), R=1-\omega_{\mathrm{e}}^{2} /\left(\omega\left(+\Omega_{\mathrm{e}}\right)\right), L=1-\omega_{\mathrm{e}}^{2} /\left(\omega\left(-\Omega_{\mathrm{e}}\right)\right)$ and $P=$ $1-\omega_{\mathrm{e}}^{2} / \omega^{2}$. Our tensor formalism as described above, however, has the advantage that it is easier to handle with the advanced symbolic tools of Mathematica.

Solutions of the dispersion relation (5) are shown in the Figs. 1-3. For parallel propagation $(\theta=0)$ the dispersion of the high-frequency modes is illustrated in Fig. 1a) using $G=2$. There exist four modes which are the Langmuir mode (Lm) at $\omega=\omega_{\mathrm{e}}$, the whistler mode (W) which resonates at
$\Omega_{\mathrm{e}}$, the L-mode (L) and the R-mode upper branch (R). With increasing wave number the last two modes become the freespace light wave branch. In order to get an impression of the modifications that may arise in the case of a kinetic treatment, an example of Vlasov dispersion analysis is presented in the lower two panels of Fig. 1 for the same parameters $(\theta=0, G=2)$, but using a finite electron plasma beta of $\beta_{\mathrm{e}}=0.002$. From Fig. $1 \mathrm{~b}$ it is visible that for all four modes the real part of the frequency is only slightly modified in comparison to the cold fluid results of Fig. 1a in regions of large wave numbers in which kinetic damping appears, as shown in Fig. 1c. The Langmuir wave (short dashed line) is weakly damped as long as $k \lambda_{D} \leq 0.1\left(\lambda_{D}\right.$ is the Debye length). The cyclotron damping of the whistler wave (solid line) becomes significant at $k c / \omega_{\mathrm{e}}>1$.

Whereas for parallel propagation $(\theta=0)$ both modes of concern, the Langmuir and the whistler wave, are completely decoupled, mixed modes with longitudinal and transverse electric field components are created at oblique propagation. Such a kind of mode connection has been discussed in previous papers on "generalized Langmuir waves" (see e.g. Willes and Cairns, 2000, and references therein) in order to explain the appearance of transverse waves for beam-excited Langmuir waves. How the dispersion characteristics change in the transition from parallel to oblique propagation, is shown in Fig. 2, where the dispersion of the (lower) three wave modes (Langmuir, whistler and L-mode) is shown for (a) $\theta=0^{\circ}$ and (b) $\theta=15^{\circ}$ taking $G=5$. The mode splitting is clearly seen and as a consequence of the merging of the whistler and Langmuir mode, the resulting mixed mode (at oblique propagation mostly called the whistler mode, Gurnett et al., 1983) has whistler wave characteristics at small wave numbers and asymptotically transitions into the Langmuir mode. A significant feature of this mode is the appearance of a phase velocity maximum at finite wave numbers $\left(k c / \omega_{\mathrm{e}}<1\right)$, which results from the different (phase velocity-wave number) dependence of both merging modes, and the associated gap above it. As known from previous studies of nonlinear stationary structures on other wave branches (multi-ion waves, whistler waves in plasmas with $G<1$ and lowerhybrid waves; see Sauer et al., 2001; Dubinin et al., 2003; Ma and Hirose, 2010), such mode characteristics are favorable for the existence of oscillitons. For obvious reasons, we use the term whistler-Langmuir oscilliton to describe the mixed mode nonlinear stationary wave structure considered in this work.

An interesting feature with respect to later discussions about main wave characteristics related to oscillitons, concerns their parameter dependence on the propagation angle $\theta$. Because of its general relevance for plasma wave emission, which has been pointed out in earlier studies by Gendrin (1961), Sauer et al. (2002) and Sydora et al. (2007), the point of maximum phase velocity is marked in this context by "GSS" to indicate the generalization of Gendrin's concept originally developed for usual whistlers in 
overdense plasmas $(G<1)$. Using the basic dispersion relation $D(\omega, k)=0$ according to Eq. (5), the frequency, wave number and (maximum) phase velocity of the mixed whistler-Langmuir mode at the GSS (generalized Gendrin) point are plotted in Fig. 3 as a function of $\theta$ for three values of the parameter $G=\Omega_{\mathrm{e}} / \omega_{\mathrm{e}}(G=10,2.5,1.3)$. As seen in the upper panel, for $G>1$ and small propagation angles $\theta$, the "oscilliton frequency" is close to the electron plasma frequency and decreases with increasing $\theta$ down to about the lower-hybrid frequency, whereas the related wave number varies between $k c / \omega_{\mathrm{e}} \sim 1$ and $k c / \omega_{\mathrm{e}} \geq 0.2$.

The angle dependence is an important signature which will be used in later discussions of plasma radiation measured by satellites in space. However, it should already be mentioned here, that the "oscilliton frequency" versus $\theta$ exhibits a clear difference compared with the prediction of the resonance cone formula (plotted in the upper panel of Fig. 3 as a dotted line; see e.g. Eq. (1) of Gurnett et al., 1983) which is normally used to explain the funnel-like wave spectra of point sources in auroral magnetospheres.

\subsection{Stationary waves}

The first step in describing oscillitons is to analyse the corresponding linear stationary waves. From the dispersion relation for high-frequency waves in a cold plasma, a phase velocity gap exists in the region between the Langmuir mode and the whistler mode, as illustrated in Fig. 2. It is in this region where complex wave numbers $\left(k=k_{\mathrm{r}}+i k_{\mathrm{i}}\right)$, representing oscillating growing solutions, should occur. To calculate $k=k(U)$, where $U$ is the velocity of the moving stationary structure, the linear dispersion relation of the cold fluid approximation $D(\omega, k)=0$ in the form of Eq. (5) is used and one replaces $\omega$ with $k U$, that means, $y \rightarrow x U$. The result is a polynomial in $z=x^{2}$ of third order

$D(x, U)=C_{0}+C_{1} z+C_{2} z^{2}+C_{3} z^{3}$

with the coefficients $C_{0}$ to $C_{3}$ given by

$C_{0}=-U^{2}$

$C_{1}=\cos ^{2} \theta+\left(2-G^{2}-G^{2} \cos ^{2} \theta\right) U^{2}+\left(G^{4}-3 G^{2}\right) U^{4}$

$C_{2}=\left(G^{2}-1\right) U^{2}+\left(4 G^{2}-2 G^{4}\right) U^{4}+\left(G^{6}-3 G^{4}\right) U^{6}$

$C_{3}=-G^{2} U^{4}+2 G^{4} U^{6}-G^{6} U^{8}$

Equation (7) represents the dispersion relation of stationary high-frequency waves which can easily be solved by standard routines. An example is shown in Fig. 4 for $G=5$ and $\theta=5^{\circ}$ where "W" marks the whistler mode and "Lm" stands for the Langmuir mode. The complex solution $k$ exists just in the phase velocity gap marked in Fig. 2 by the shaded strip. It is caused by the coupling between the whistler and the Langmuir mode at finite propagation angle. Correspondingly, the imaginary part of $k$ disappears if $\theta$ goes to zero. The dotted curve in the $k_{\text {i }}$ plot (lower panel of Fig. 4) belongs to the phase velocity maximum of the whistler mode at $k c / \omega_{\mathrm{e}}>1$,
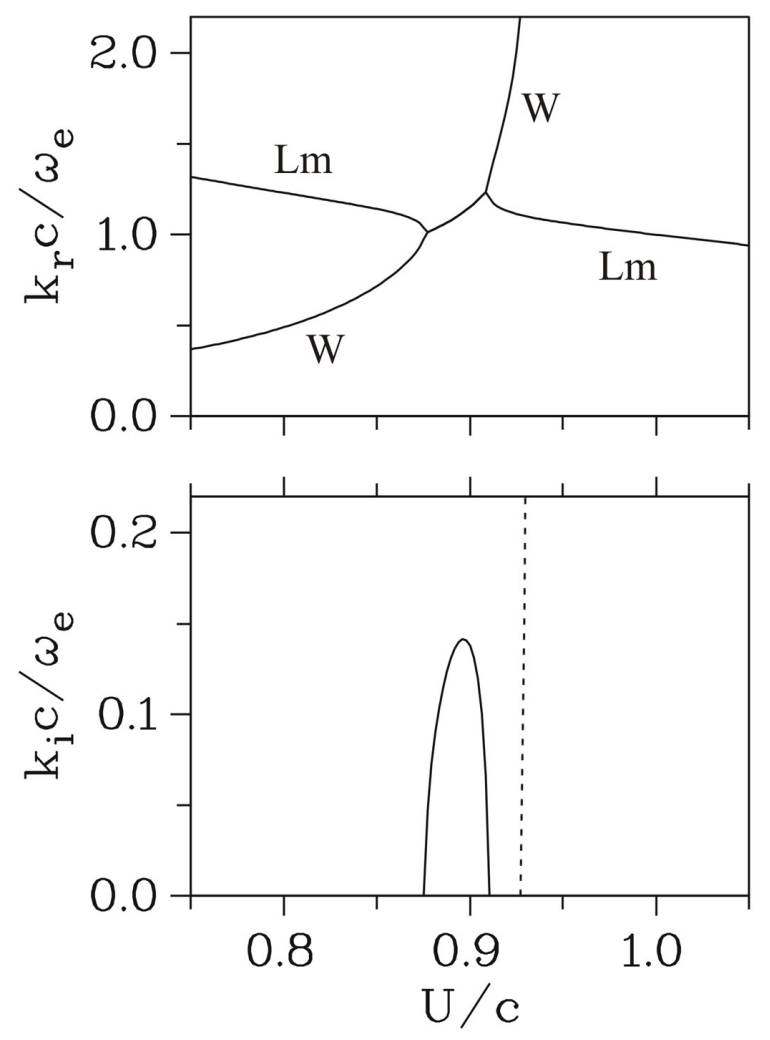

Fig. 4. Wave number (real and imaginary part) versus "oscilliton velocity" $U$ resulting from the coupling between Langmuir $(\mathrm{Lm})$ and whistler mode (W) at oblique propagation; $G=5, \theta=5^{\circ}$. Around $U / c \sim 0.9$ growing oscillating waves (oscillitons) may exist.

like in a plasma with $\mathrm{G}<1$ (see Fig. 7 in Sauer and Sydora, 2010), and will not further be considered here.

\section{Whistler-Langmuir oscillitons}

The procedure to calculate the spatial profiles of whistlerLangmuir oscillitons as a particular type of stationary nonlinear structure in a magnetized electron-proton plasma with $G=\Omega_{\mathrm{e}} / \omega_{\mathrm{e}}>1$ is the same as previously described in other papers on oscillitons, especially on whistler oscillitons in overdense plasmas $(G<1)$; e.g. Sauer et al., 2002; Dubinin et al., 2003; Cattaert and Verheest, 2005). Since whistlerLangmuir oscillitons arise from the coupling between two different wave modes whereby one of them, the Langmuir mode, is mainly electrostatic because of charge separation, the Poisson equation and the displacement current are essential terms that have to be taken into account.

As is common in soliton theory, the analysis of WL oscillitons is carried out in a slab model where all parameters only depend on the $\mathrm{x}$-coordinate. Here, the plasma forms the rest frame in which the structures move with the velocity $U$ in the $\mathrm{x}$-direction. The undisturbed magnetic field lies in the $\mathrm{x}-\mathrm{z}$ plane, inclined by the angle $\theta$ relative to the 


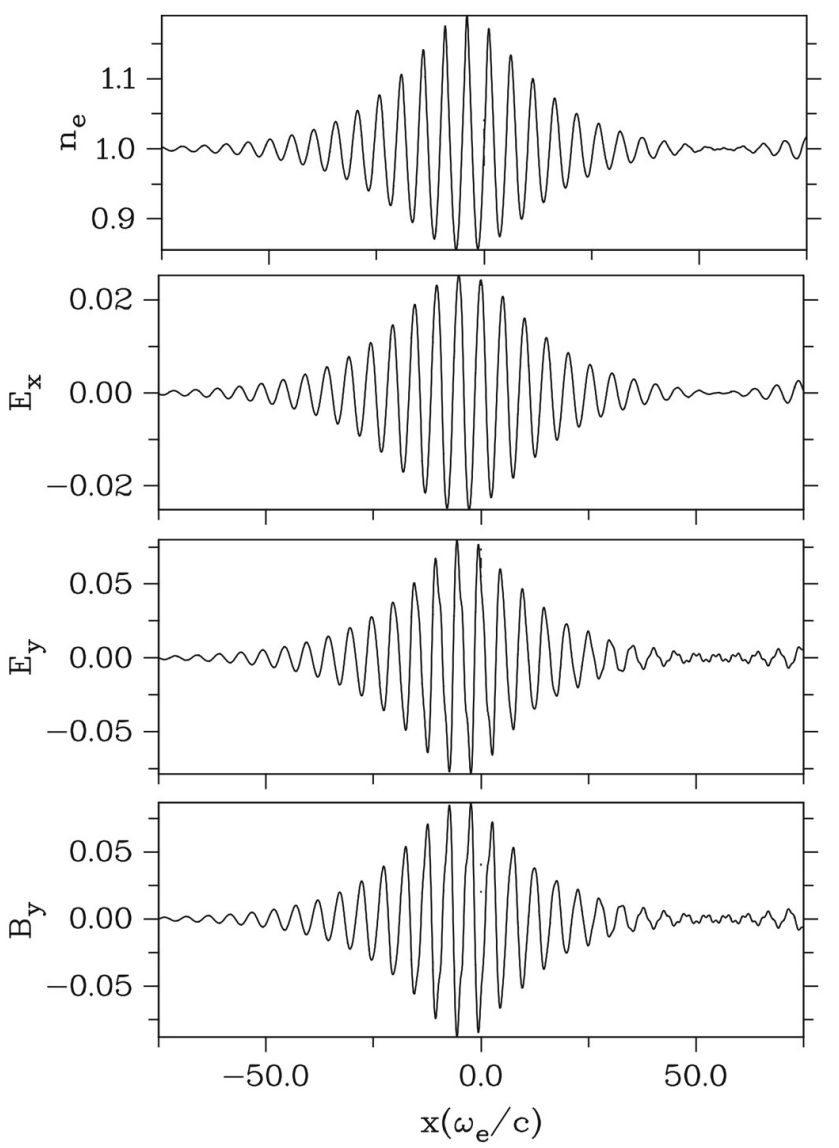

Fig. 5. Spatial profiles of a whistler-Langmuir oscilliton taking $G=$ 5 and $\theta=5^{\circ}$; from top to bottom: electron density normalized to $n_{\mathrm{eo}}, \mathrm{x}$ - and y-component of the electric field in units of $E_{0}=c B_{0}$ and the y-component of the magnetic field normalized to $B_{0}$. The oscilliton velocity was selected as $M=U / c=0.9085$.

$\mathrm{x}$-axis, $B=B_{0}(\cos \theta, 0, \sin \theta)$. No thermal effects are included. Vlasov dispersion theory verifies the cold plasma assumption since kinetic damping in the wave number range of interest $\left(k c / \omega_{\mathrm{e}} \leq 1\right)$ of both the Langmuir and the whistler wave mode is weak as long as for moderate values of $\mathrm{G}$ the electron plasma beta remains small enough $\left(\beta_{\mathrm{e}} \leq 0.02 / G^{2}\right)$.

Analysis of stationary solutions to the magnetized fluidMaxwell equations in which the time dependence appears only in the form $f(x-U t)$ leads us to the following set of equations, written in normalized form as:

$\frac{d v_{\mathrm{e}, \mathrm{px}}}{d x}=\frac{q_{\mathrm{e}, \mathrm{p}}}{\mu_{\mathrm{e}, \mathrm{p}}}\left(E_{\mathrm{x}}+v_{\mathrm{e}, \mathrm{py}} B_{\mathrm{z}}-v_{\mathrm{e}, \mathrm{pz}} B_{\mathrm{y}}\right) /\left(M-v_{\mathrm{e}, \mathrm{px}}\right)$

$\frac{d v_{\mathrm{e}, \mathrm{py}}}{d x}=\frac{q_{\mathrm{e}, \mathrm{p}}}{\mu_{\mathrm{e}, \mathrm{p}}}\left(E_{\mathrm{y}}-v_{\mathrm{e}, \mathrm{px}} B_{\mathrm{z}}+v_{\mathrm{e}, \mathrm{pz}} B_{\mathrm{x}}\right) /\left(M-v_{\mathrm{e}, \mathrm{px}}\right)$

Equations of motion for electrons and protons:

$\frac{d v_{\mathrm{e}, \mathrm{pz}}}{d x}=\frac{q_{\mathrm{e}, \mathrm{p}}}{\mu_{\mathrm{e}, \mathrm{p}}}\left(E_{\mathrm{z}}+v_{\mathrm{e}, \mathrm{px}} B_{\mathrm{y}}-v_{\mathrm{e}, \mathrm{py}} B_{\mathrm{x}}\right) /\left(M-v_{\mathrm{e}, \mathrm{px}}\right)$
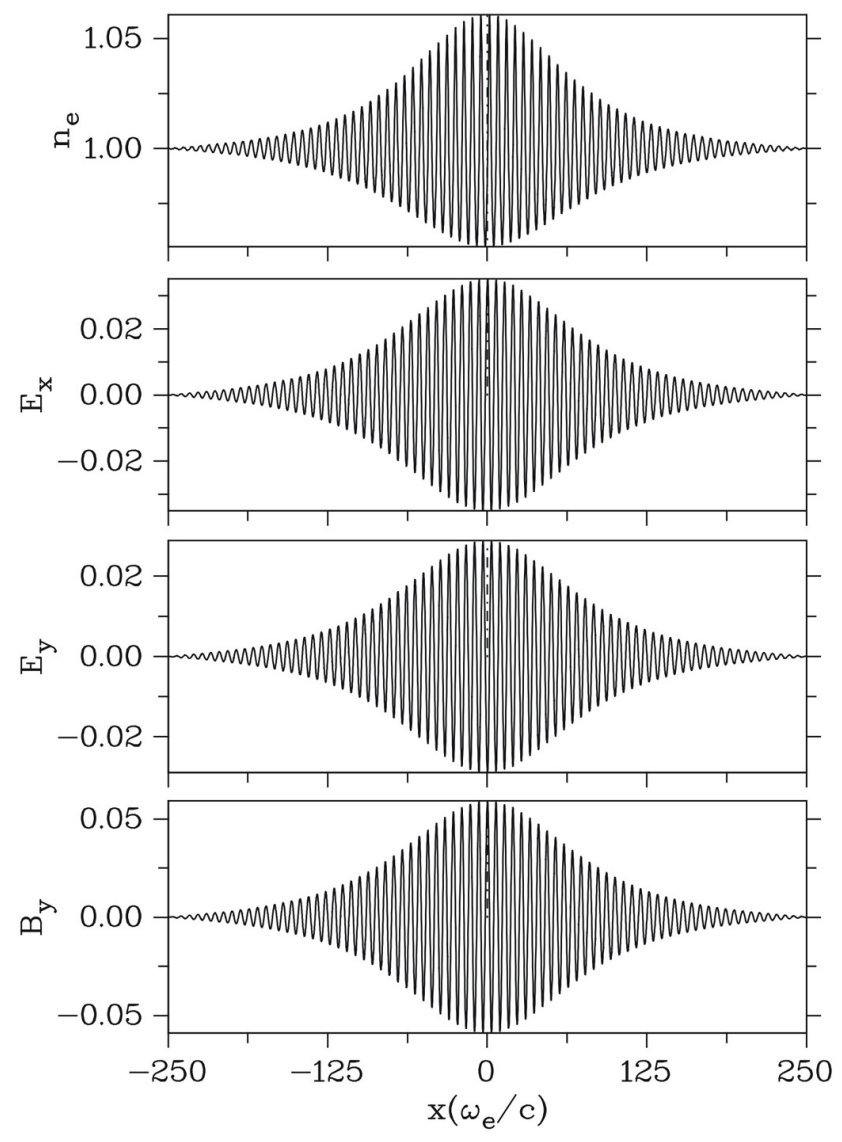

Fig. 6. Spatial profiles of a WL oscilliton, as in Fig. 5. Here, the parameters are $G=1.5$ and $\theta=30^{\circ}$. The oscilliton velocity was selected as $M=0.48965$.

Continuity equations:

$n_{\mathrm{p}}=\frac{n_{p 0} M}{\left(M-v_{\mathrm{px}}\right)} \quad ; \quad n_{\mathrm{e}}=\frac{n_{e 0} M}{\left(M-v_{\mathrm{ex}}\right)}$

Ampere's law:

$\frac{d B_{\mathrm{y}}}{d x}=+\frac{n_{\mathrm{p}} v_{\mathrm{pz}}-n_{\mathrm{e}} v_{\mathrm{ez}}}{\left(1-M^{2}\right) G} \quad ; \quad \frac{d B_{\mathrm{y}}}{d x}=-\frac{n_{\mathrm{p}} v_{p y}-n_{\mathrm{e}} v_{\mathrm{ey}}}{\left(1-M^{2}\right) G}$

Poisson equation:

$\frac{d E_{\mathrm{x}}}{d x}=\left(n_{\mathrm{p}}-n_{\mathrm{e}}\right) / G$

Faraday equation:

$E_{\mathrm{y}}=M\left(B_{\mathrm{z}}-B_{\mathrm{z} 0}\right) \quad ; \quad E_{\mathrm{z}}=-M B_{\mathrm{y}}$

The spatial coordinate $x$ is normalized to the collisionless electron skin depth $\left(x \rightarrow x \omega_{\mathrm{e}} / c\right)$. M is the oscilliton velocity normalized to $c$, the speed of light, $M=U / c$ and correspondingly, all velocities are normalized to $c$. The electric field is in units of $E_{0}=c B_{0}$ where $B_{0}$ is the undisturbed magnetic field value. $\mu_{\mathrm{e}, \mathrm{p}}$ is the relative mass (in units of the electron mass $\left.m_{\mathrm{e}}\right), \mu_{\mathrm{e}}=1, \mu_{\mathrm{p}}=m_{\mathrm{p}} / m_{\mathrm{e}} ; q_{\mathrm{e}, \mathrm{p}}$ is the electric charge 
with $q_{\mathrm{e}}=-1, q_{\mathrm{p}}=+1$. Relativistic corrections in the equations of motion (see e.g. McKenzie et al., 2005) are not considered here since all velocities remain small compared with $c(v / c \leq 0.1)$. The system of Eqs. (8)-(14) admits the following constants of motion (momentum flux conservation), (e.g. McKenzie et al., 2004, 2005).

$$
\begin{aligned}
& M\left(\mu_{\mathrm{e}} v_{\mathrm{ex}}+\mu_{\mathrm{p}} v_{\mathrm{px}}\right)+0.5\left(\left(B^{2}-1\right)+E_{\mathrm{x}}^{2} G^{2}\right)=0 \\
& M\left(\mu_{\mathrm{e}} v_{\mathrm{ey}}+\mu_{\mathrm{p}} v_{\mathrm{py}}\right)+B_{\mathrm{x}} B_{\mathrm{y}}+E_{\mathrm{x}} E_{\mathrm{y}} G=0 \\
& M\left(\mu_{\mathrm{e}} v_{\mathrm{ez}}+\mu_{\mathrm{p}} v_{\mathrm{pz}}\right)+B_{\mathrm{x}} B_{\mathrm{z}}+E_{\mathrm{x}} E_{\mathrm{z}} G=0
\end{aligned}
$$

Therefore, the magnetic field components, for example, can be expressed as a function of the velocity and electric field components.

To find oscilliton-type solutions of the system of equations given above, for a chosen set of parameters $G$ and $\theta$, one has to select an "oscilliton speed" $M$ for which, from linear stationary theory, growing solutions are predicted. As an initial disturbance, only for the transverse electron velocity, a finite value $v_{\mathrm{ez}} \ll 1$ in the range $10^{-5}-10^{-4}$ was taken. All other disturbances were set to zero. For such initial conditions the resulting spatial profiles have almost the same amplitude and width, which is a characteristic signature of solitary structures. Using the parameters of Fig. $4\left(G=5, \theta=5^{\circ}\right)$, spatial profiles of a whistler-Langmuir oscilliton are shown in Fig. 5. It has the typical wave packet structure with spatial oscillation wavelength approximately $\lambda \sim 6 c / \omega_{\mathrm{e}}$, in agreement with the predictions of Fig. 4 where the maximum of $k_{\mathrm{i}}$ appears at $k_{\mathrm{r}} c / \omega_{\mathrm{e}} \sim 1$. Since the wave packet has a characteristic extension of about $100 c / \omega_{\mathrm{e}}$, for a phase velocity close to $c$, the wave packet repetition time is about $100 \omega_{\mathrm{e}}^{-1}$.

Figure 6 shows another example of a more extended whistler-Langmuir oscilliton using $G=1.5$ and $\theta=30^{\circ}$. Using Fig. 3, it is easy to determine that the corresponding oscilliton velocity decreases to $M \sim 0.5$. Its chosen, exact value is $M=0.48965$. For these parameters, the WL wave packet has an extension of about $500 c / \omega_{\mathrm{e}}$, five times more than in the previous case. The wave length of the spatial oscillitons is similar as before determined by $k c / \omega_{\mathrm{e}} \sim 1$.

\section{Beam-excited whistler-Langmuir waves}

Before presenting particle simulations of beam-excited whistler-Langmuir waves, their regime of linear instability is studied by means of fluid dispersion analysis. For our situation of interest, with wave numbers in the range $k c / \omega_{\mathrm{e}} \sim 1$, cold theory is a sufficient approximation as a first step. This is because of the corresponding small values of $k \lambda_{D}=\left(k c / \omega_{\mathrm{e}}\right)\left(v_{\mathrm{e}} / c\right)$; as long as the electron thermal velocity $v_{\mathrm{e}}$ is small compared to $\mathrm{c}$ this allows us to neglect Landau damping. The procedure to derive the dispersion relation $D(\omega, k)=0$ follows the same steps as described above, but adding a second equation of motion for the beam electrons and a corresponding contribution to the current into the

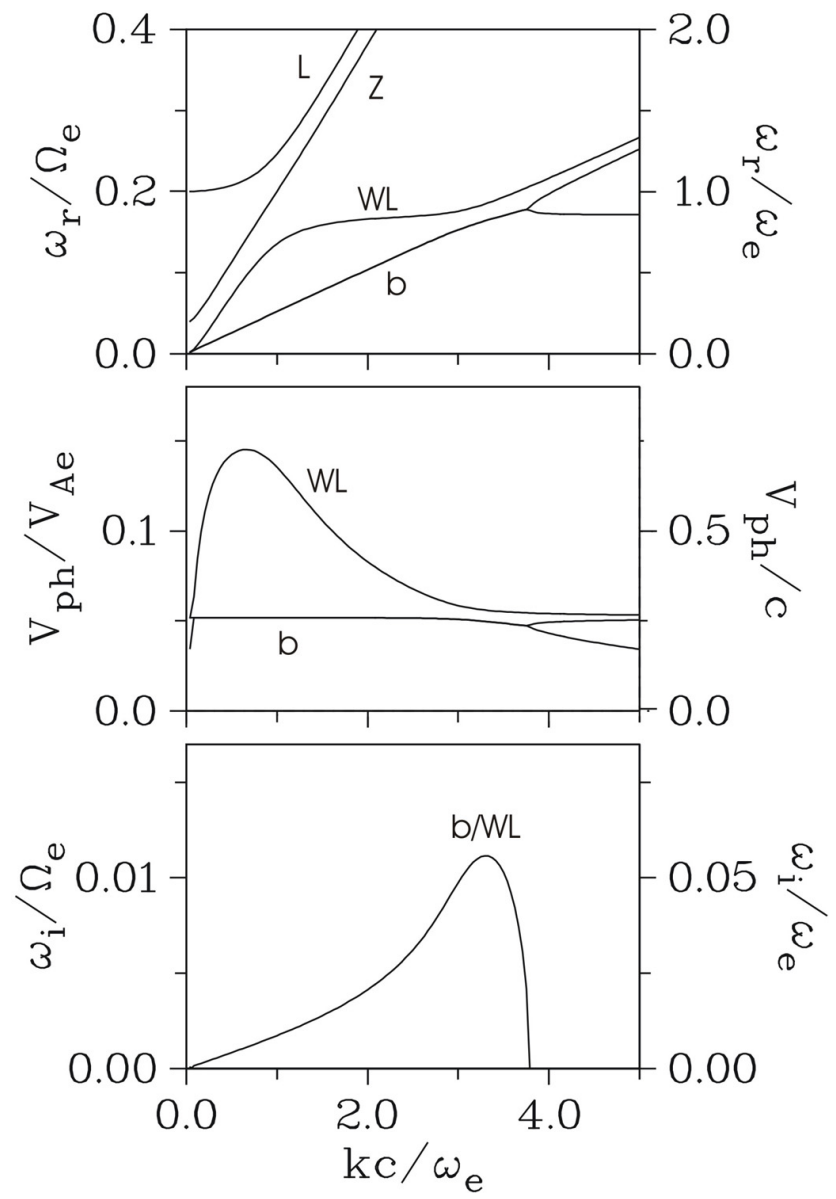

Fig. 7. Excitation of whistler-Langmuir waves by an electron beam and related dispersion of the three wave modes in the vicinity of the electron plasma frequency $\omega_{\mathrm{e}}$ for an (underdense) plasma with $G=\Omega_{\mathrm{e}} / \omega_{\mathrm{e}}=5$ at oblique propagation, $\theta=30^{\circ}$, see also Fig. 2 . The beam density is $n_{\mathrm{b}} / n_{o}=0.001$ and beam velocity $V_{\mathrm{b}} / c=0.3$. The WL mode becomes unstable and has its maximum growth rate at $k c / \omega_{\mathrm{e}} \sim 3.3$, which follows from the resonance condition $k V_{\mathrm{b}} \sim$ $\omega_{\mathrm{e}}$. The notation "b/WL" should indicate that the instability arises from the interaction of the beam (b) with the whistler-Langmuir (WL) mode.

Maxwell equation; see also Sauer and Sydora (2010). The electron beam is assumed to move parallel to the magnetic field with a velocity $V_{\mathrm{b}}$. The resulting dispersion relation can now be written as a polynomial of 12-th order in $\omega$ or $y$, respectively.

A representative example for the dispersion of beamexcited WL waves, using $G=5$ and $\theta=30^{\circ}$, is shown in Fig. 7 for a beam with a density of $n_{\mathrm{b}} / n_{o}=0.001$ and a velocity of $V_{\mathrm{b}} / c=0.3$. The instability is of Cerenkov-type and arises from resonant interaction of the beam mode $\omega=$ $k V_{\mathrm{b}} \cos \theta$ with the mostly electrostatic part of the whistlerLangmuir mode, very similar to beam-plasma interaction in isotropic plasmas. The maximum growth rate appears at $k c / \omega_{\mathrm{e}} \sim\left(V_{\mathrm{b}} / c\right)^{-1}$. 


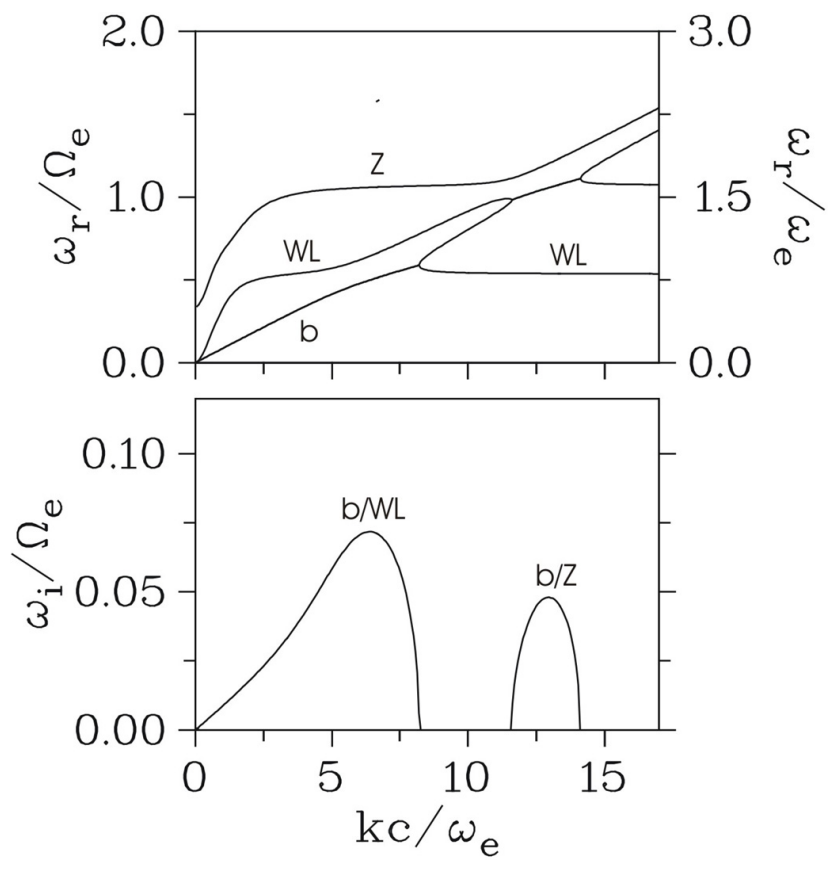

Fig. 8. Excitation of both modes, the whistler-Langmuir mode and the Z-mode $\left(G=1.5, \theta=30^{\circ}\right)$, by an electron beam with parameters $n_{\mathrm{b}} / n_{o}=0.01, V_{\mathrm{b}} / c=0.15$. The notations "b/WL" and "b/Z" should indicate the different origins of both instabilities.

For the case that both modes, the whistler-Langmuir mode and the Z-mode, are not so largely separated, this means $G \sim 1$, and simultaneous excitation by one and the same electron beam is possible. Such a situation is shown in Fig. 8 using $G=1.5$. The beam parameters are $n_{\mathrm{b}} / n_{o}=0.01, V_{\mathrm{b}} / c=$ 0.15 . The second maximum of instability at $k c / \omega_{\mathrm{e}}>10$ is due to Cerenkov interaction with the Z-mode at $\omega \sim \Omega_{\mathrm{e}}$. Because of the large wave number, however, it may happen that the growth rate is strongly reduced and, possibly, the second instability is completely annihilated if Landau damping effects are taken into account.

Next, modifications of the whistler-Langmuir wave dispersion in thermal plasmas are discussed, which occur if resonant wave-particle interaction begin to play a role. From the relation $v_{\mathrm{e}} / c=G\left(\beta_{\mathrm{e}} / 2\right)^{-1}$ and $G>1$, this may happen in plasmas with relatively low electron beta. One example of how the beam instability is affected by finite electron temperature effects $\left(\beta_{\mathrm{e}}=0.001\right)$ is shown in Fig. 9 using Vlasov dispersion theory. As shown in the figure, the most pronounced effect is the kinetic damping of the beam mode itself which leads to a reduced growth rate of the instability compared with cold plasma conditions. With increasing electron plasma beta an increasing beam density is necessary to compensate the enhanced damping.

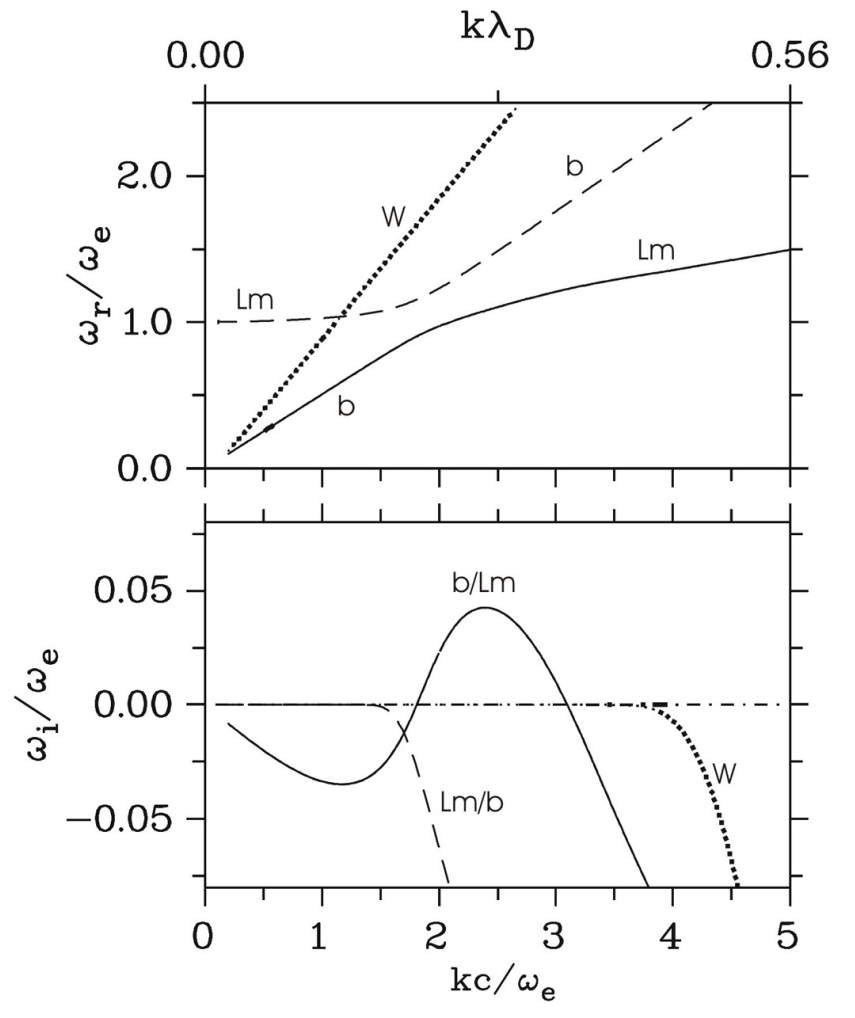

Fig. 9. Dispersion of beam-excited Langmuir waves, Vlasov approach: Real (top panel) and imaginary part of frequency (bottom panel) versus wave number $k$ (all in normalized units) as result of Vlasov dispersion theory. The parameters are: $\theta=0^{\circ}$ (parallel propagation), $G=5, \beta_{\mathrm{e}}=0.001, n_{\mathrm{b}} / n_{o}=0.005, V_{\mathrm{b}} / c=0.5$ and $T_{\mathrm{b}} / T_{\mathrm{e}}=0.5$. The dotted line marks the whistler mode which is nearly undamped within the considered wave number range. The scale on top gives the wave number in units of the reciprocal Debye length $\lambda_{D}$ according to $k \lambda_{D}=G\left(k c / \omega_{\mathrm{e}}\right)\left(\beta_{\mathrm{e}} / 2\right)^{1 / 2}$.

\section{Kinetic simulations of beam-excited waves}

Fully electromagnetic particle-in-cell (PIC) simulations (1$\mathrm{D}$ real space and 3-D velocity space) have been carried out using the model described in our previous study of parallel propagating whistler instability driven by electron temperature anisotropy (Sydora et al., 2007). The background magnetic field lies in the $\mathrm{X}-\mathrm{z}$ plane, inclined by the angle $\theta$ relative to the $\mathrm{x}$-axis such that $\boldsymbol{B}=B_{0}(\cos \theta, 0, \sin \theta)$. Relativistic effects are included in the equations of motion for the charged particles. Three species of plasma charged particles are included in the simulations: electron beam $\left(n_{\mathrm{b}}\right)$, background electrons $\left(n_{o}\right)$ and ions. The plasma is assumed to be cold and a realistic proton to electron mass ratio $\left(m_{\mathrm{i}} / m_{\mathrm{e}}=1837\right)$ used. The boundary condition on the particles and fields in the $\mathrm{x}$-direction were taken to be periodic. Particles are loaded uniformly in the $\mathrm{x}$-direction and approximately 100 particles per cell were used for each species. The number of cells was taken as $L_{\mathrm{X}}=1024 \Delta$ with cell size 


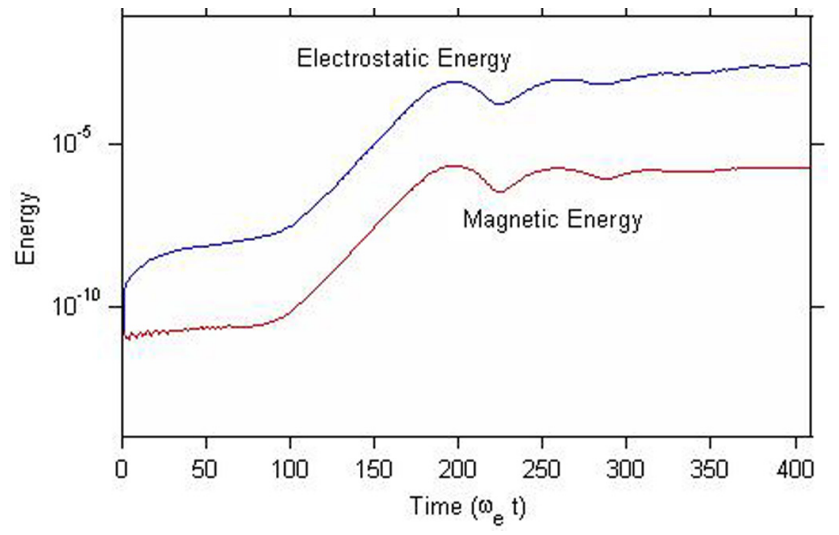

Fig. 10. Temporal evolution of the electrostatic (upper blue curve) and magnetic energy (red curve). The parameters used in the simulation are: $G=5, \theta=5^{\circ}, n_{\mathrm{b}} / n_{o}=0.002, V_{\mathrm{b}} / c=0.5$. Saturation of both components occurs at about $\omega_{\mathrm{e}} t=180$.

$\Delta=0.1 c / \omega_{\mathrm{e}}$. The time step $\omega_{\mathrm{e}} \Delta t=0.05$ was selected to ensure the Courant condition $(c \Delta t / \Delta x<1)$ for light waves is satisfied.

The parameters chosen are similar to that of Fig. 7, but with $\theta=5^{\circ}$ and a larger beam velocity: $G=5, n_{\mathrm{b}} / n_{o}=$ $0.002, V_{\mathrm{b}} / c=0.5$. A higher beam velocity was chosen in order to reduce the resonant wave number which follows from the relation $k c / \omega_{\mathrm{e}} \sim c / V_{\mathrm{b}}$. As a result, Fig. 10 shows the temporal evolution of both the electrostatic and magnetic energy. After the end of the linear growth phase owing to resonant beam-plasma interaction, at about $\omega_{\mathrm{e}} t=180$, the system begins to saturate and remains at nearly the same energy level. Because of the small propagation angle and the associated dominance of electrostatic (Langmuir) waves, it is not surprising that the electrostatic energy exceeds its magnetic counterpart by more than two orders of magnitude.

Interesting characteristics of the beam-plasma instability and the subsequent nonlinear saturation become visible in Fig. 11 where the temporal evolution of the magnetic field component $B_{\mathrm{y}}$ is plotted versus the wave number $k c / \omega_{\mathrm{e}}$ together with the linear dispersion of the relevant wave modes in the frequency range close to the electron plasma frequency. As predicted, maximum growth rate appears at $k c / \omega_{\mathrm{e}} \sim 2.2$. As further observed in the figure, the instability saturates at about $\omega_{\mathrm{e}} t=180$. In this context, a remarkable signature is certainly the occurrence of a second maximum of $B_{\mathrm{y}}$ in the vicinity of $k c / \omega_{\mathrm{e}}=1$ at nearly the same time. Consistent with our preceding studies on the physical meaning of generalized Gendrin modes, we suggest that these waves represent whistler-Langmuir oscillitons. Obviously, they arise from the accumulation of waves at the point where phaseand group velocity coincide.

Altogether, we physically describe the following process of beam-plasma interaction: (1) large-amplitude electrostatic waves are generated by resonant Cerenkov interaction.

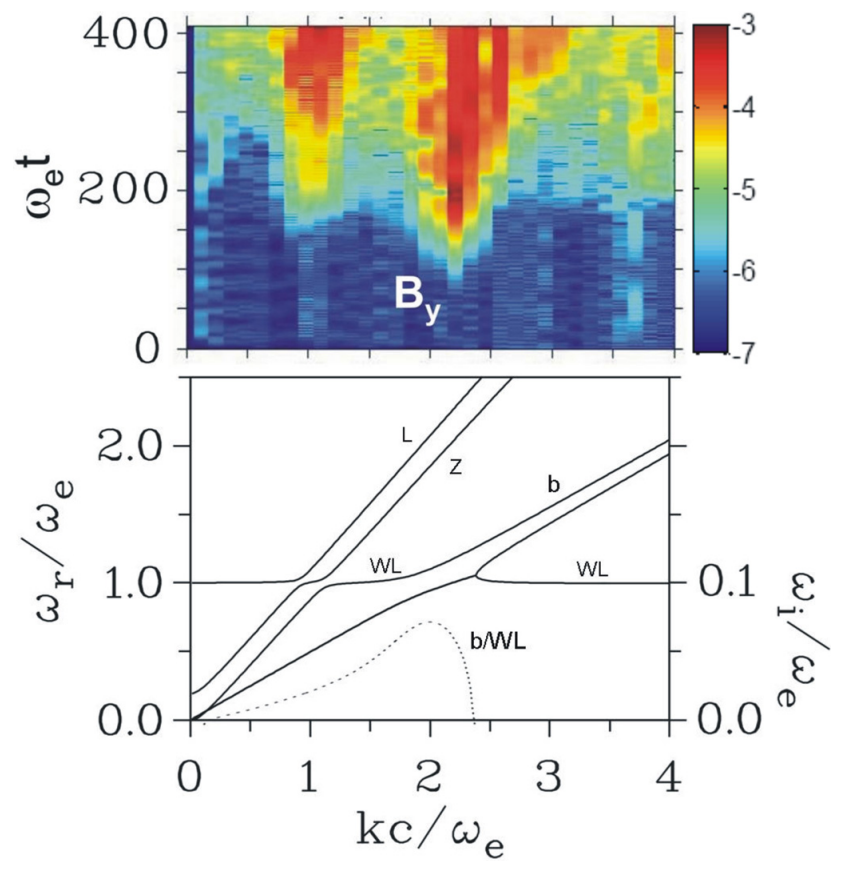

Fig. 11. Temporal evolution of the magnitude of the magnetic field component $B_{\mathrm{y}}$ (logarithmic scale) versus wave number (on top) and related linear dispersion (on bottom). Solid curves represent the real part of frequency; the dotted curve indicates the growth rate. The maximum intensity of $B_{\mathrm{y}}$ at $k c / \omega_{\mathrm{e}} \sim 2.2$ in the upper color plot results from resonant beam-plasma interaction. At $\omega_{\mathrm{e}} t \geq 200$, a second maximum appears near $k c / \omega_{\mathrm{e}}=1$ and begins the accumulation of wave energy at the Gendrin point which is related to the formation of whistler-Langmuir oscillitons.

(2) The subsequent saturation process is accompanied by the nonlinear excitation of waves whose spectrum shifts during their temporal evolution more and more to shorter wave numbers. (3) If a generalized Gendrin point exists in that range of wave numbers, the waves may accumulate around this point, finally forming stationary nonlinear structures there. In our particular case, that means the occurrence of whistlerLangmuir oscillitons. The described process of nonlinear wave generation in the whistler-Langmuir frequency range is also evident from Fig. 12 in which the $(\omega, k)$ spectrum of both the electric and magnetic field, calculated over the whole time interval, is shown. Whereas the spectrum of the electric field reflects predominately the source region around $k c / \omega_{\mathrm{e}} \sim 2.2$, the shift of the magnetic field spectrum to the Gendrin point $\left(k c / \omega_{\mathrm{e}} \sim 1\right)$, where the wave has mainly electromagnetic character, is clearly visible. The ratio $c B / E$ is close to unity there. Since waves with $k c / \omega_{\mathrm{e}} \sim 1$ are generally weakly damped, Gendrin mode waves, once generated somewhere, may propagate over large distances without any great dissipation.

Therefore, the PIC simulations have demonstrated that one has to distinguish between two types of waves which may be essential for the interpretation of hiss phenomena in 


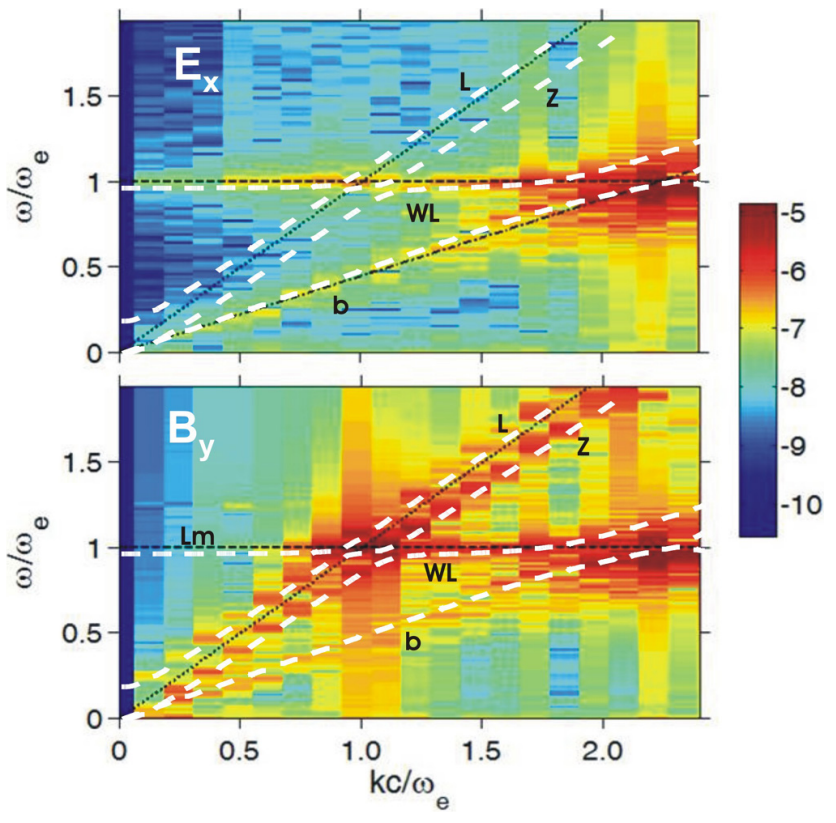

Fig. 12. $(\omega, k)$ spectrogram of the electric (on top) and magnetic field components (on bottom). Color scale indicates logarithmic magnitude. The dashed white lines represent the dispersion curves from fluid theory including the beam. The dotted black line marks the light curve, $\omega / k=c$, which for $\omega>\omega_{\mathrm{e}}$ is nearly identical with the L mode. Maximum electric and magnetic field intensity appear at the intersection of the whistler-Langmuir mode WL $\left(\omega \sim \omega_{\mathrm{e}}\right)$ with the Cerenkov beam mode $\mathrm{b}\left(\omega \sim k V_{\mathrm{b}}\right)$. A second maximum of magnetic energy appears at the Gendrin point near $k c / \omega_{\mathrm{e}}=1$, $\omega \sim \omega_{\mathrm{e}}$

magnetospheric plasmas. One occurs in the source region and is directly related to the beam-plasma instability, which generates mostly electrostatic waves with relative large wave number, $k c / \omega_{\mathrm{e}} \geq 5$, depending on the beam velocity. The other is associated with whistler-Langmuir oscillitons as a class of stationary nonlinear waves with $k c / \omega_{\mathrm{e}} \sim 1$ which may form away from the source as consequence of (nonlinear) wave number shift from the range of unstable waves to the Gendrin point where phase- and group velocity coincide.

\section{Discussion and summary}

The main focus of this paper was directed towards the description of whistler-Langmuir oscillitons as a new type of solitary structure existing in plasmas in which the electron cyclotron frequency $\left(\Omega_{\mathrm{e}}\right)$ is larger than or comparable to the electron plasma frequency, i.e. $G=\Omega_{\mathrm{e}} / \omega_{\mathrm{e}} \geq 1$. Another aspect was to show how they arise and to reveal their physical relevance in interpreting plasma radiation in space, especially auroral hiss in planetary magnetospheres. The property $G \geq 1$ has the consequence that both the whistler and Langmuir wave, which intersect at parallel propagation as independent modes, become coupled and develop into mixed modes for propagation oblique to the background magnetic field. On the other hand, this mode coupling, leads to a particular dispersion of the lower mode (here called the whistlerLangmuir mode) which is characterized by the occurrence of a Gendrin point where the phase- and group velocity coincide. Finally, this provides the requirement for the existence of stationary waves with growing oscillating structure which, in our particular case, means the existence of whistler-Langmuir oscillitons.

Evidence of nonlinear stationary structures represents an important first step in plasma theory. But, it remains incomplete if the problem concerning their driving mechanism is not addressed. Since Langmuir waves can easily be excited by beam-plasma instability, it is apparent that this process is also the dominant source of whistler-Langmuir radiation. In contrast to the purely electrostatic Langmuir waves well studied in past, analogous investigations in magnetized plasmas at oblique propagation, however, require the full apparatus of electromagnetic plasma dispersion theory and less work on this has appeared in the literature (see e.g. Kennel, 1966; Maggs, 1976; Lin et al., 1984; Morgan et al., 1994; Willes and Cairns, 2000; Kopf et al., 2010).

Our investigation consisted of two parts. In the first part, we consistently applied the magnetized fluid-Maxwell theory to both the: (i) full electromagnetic dispersion analysis of beam-excited whistler-Langmuir waves and (ii) studies on whistler-Langmuir oscillitons as a particular type of stationary nonlinear wave. In the second part, particle-in-cell (PIC) simulations have been performed to understand how the two aspects of the fluid approach (beam-plasma instability and oscillitons) remain in relation to the evolution of the beamplasma instability and its nonlinear saturation.

There is clear evidence that the beam-instability in plasmas with $G>1$ proceeds in the following steps: in the linear stage, mostly electrostatic (Langmuir) waves are excited in the wave number range of $k c / \omega_{\mathrm{e}} \sim c / V_{\mathrm{b}}$ where $V_{\mathrm{b}}$ is the beam speed. Since the frequency at large wave numbers and propagation angle are related by the resonance cone condition, this explains why a funnel-like spectrum is a typical feature of plasma radiation originating in the source region where electron beams are present (Gurnett et al., 1983). The subsequent process of nonlinear saturation of the beam-plasma instability is characterized by the excitation of waves whose spectral maximum shifts in time to smaller and smaller wave numbers up to the Gendrin point where the phase velocity reaches its maximum. Here, an accumulation of wave energy in the form of whistler-Langmuir oscillitons takes place. Because the oscilliton-related wave numbers are $k c / \omega_{\mathrm{e}} \sim 1$, the corresponding waves are only weakly damped and so may propagate over large distances without great loss.

The observed wave number shift from large to small values seems to be a more general feature of nonlinear plasma dynamics, completely different from linear processes owing to density gradients as considered, for example, by Morgan 

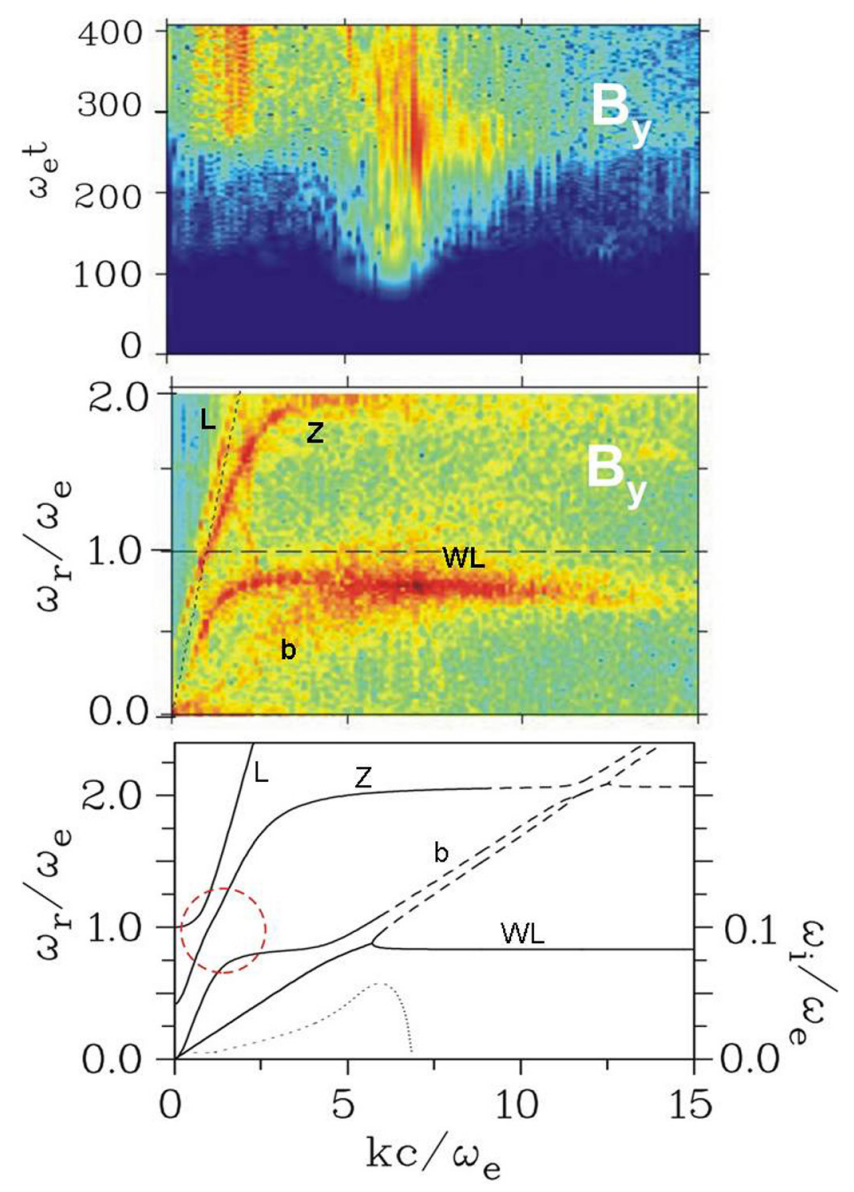

Fig. 13. Temporal evolution of the magnitude of the magnetic component $B_{\mathrm{y}}$ versus wave number $k c / \omega_{\mathrm{e}}$ (upper panel) and $(\omega, k)$ spectrum of $B_{\mathrm{y}}$ (middle panel) from PIC simulations. The parameters are: $\Omega_{\mathrm{e}} / \omega_{\mathrm{e}}=2, \theta=30^{\circ}, n_{\mathrm{b}} / n_{0}=0.005, V_{\mathrm{b}} / c=0.15$. Lower panel: Fluid dispersion of beam-excited whistler-Langmuir waves: real $\left(\omega_{\mathrm{r}}\right)$ and imaginary part of frequency $\left(\omega_{\mathrm{i}}\right)$ versus wave number. The dotted curve represents the growth rate. Dashed lines on bottom panel indicate strong damping appearing in case of kinetic treatment.

et al. (1994). It has also been found in simulations including other wave modes, e.g. whistler waves in plasmas with $G<1$ driven by temperature anisotropy (Sydora et al., 2007; Schriver et al., 2010) and electromagnetic ioncyclotron (EMIC) waves in single- and multi-ion plasmas (Silin et al., 2011; Usanova, 2010). In a recent study by Schriver et al. (2010), which is directly relevant for whistler emission in the inner magnetosphere, 2-D particle-in-cell simulations have been carried out showing that the linearly stable wave modes below $0.5 \Omega_{\mathrm{e}}$ are nonlinearly excited by wave-wave coupling between two different wave modes of the unstable spectrum.

It seems that the concept of nonlinear wave number shift has important implications for radiation problems. For example, following the paper by Morgan et al. (1994), stan-

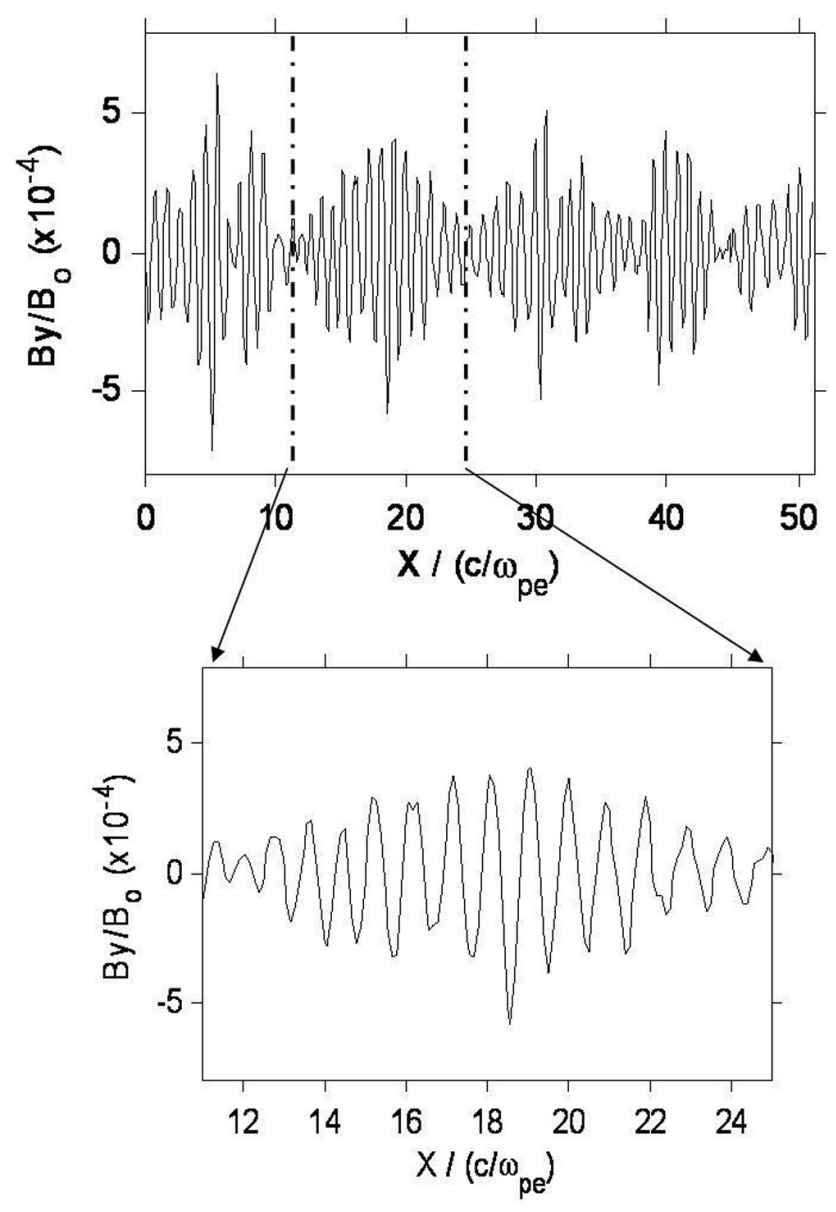

Fig. 14. Spatial profile of the magnetic field component $B_{\mathrm{y}}$ at $\omega_{\mathrm{e}} t=400$ for the same run as in Fig. 13. The lower plot shows a wave packet with an extension of about $12 c / \omega_{\mathrm{e}}$ which consists of the short-scale beam-excited wave with a wave length of $\lambda \sim 0.9 c / \omega_{\mathrm{e}}$, in good agreement with the value following from the resonance condition $k c / \omega_{\mathrm{e}} \sim c / V_{\mathrm{b}}\left(V_{\mathrm{b}} / c=0.15\right)$. The average extension of the wave packet itself is well related to the wave number of the corresponding Gendrin mode, $k c / \omega_{\mathrm{e}}<1$; see Fig. 3 .

dard models of auroral hiss generation and propagation suffer from the obvious discrepancy between strong Landau damping of whistler-Langmuir waves at large wave numbers within the source region and the observational fact that auroral hiss may propagate over large distances. Keeping in mind, however, the PIC simulation results shown in Figs. 11 and 13, after which spectral shift to smaller wave numbers is an accompanying fundamental element of nonlinear wave saturation, the above discrepancy finds a natural solution.

Another point that should be mentioned concerns the occasional observation of plasma radiation above the local plasma frequency as discussed in the paper by Kopf et al. (2010) for auroral hiss at Saturn. They argue that such emission is not allowed and try to explain the observed effect by Doppler shift owing to spacecraft and plasma motions combined with 
Nonlinear wave
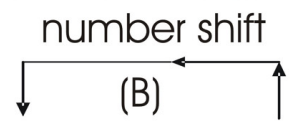

Region of stationary Source region: nonlinear waves: oscillitons beam-plasma

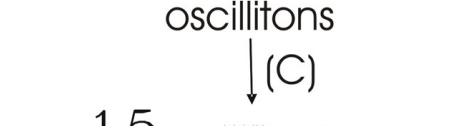
instability
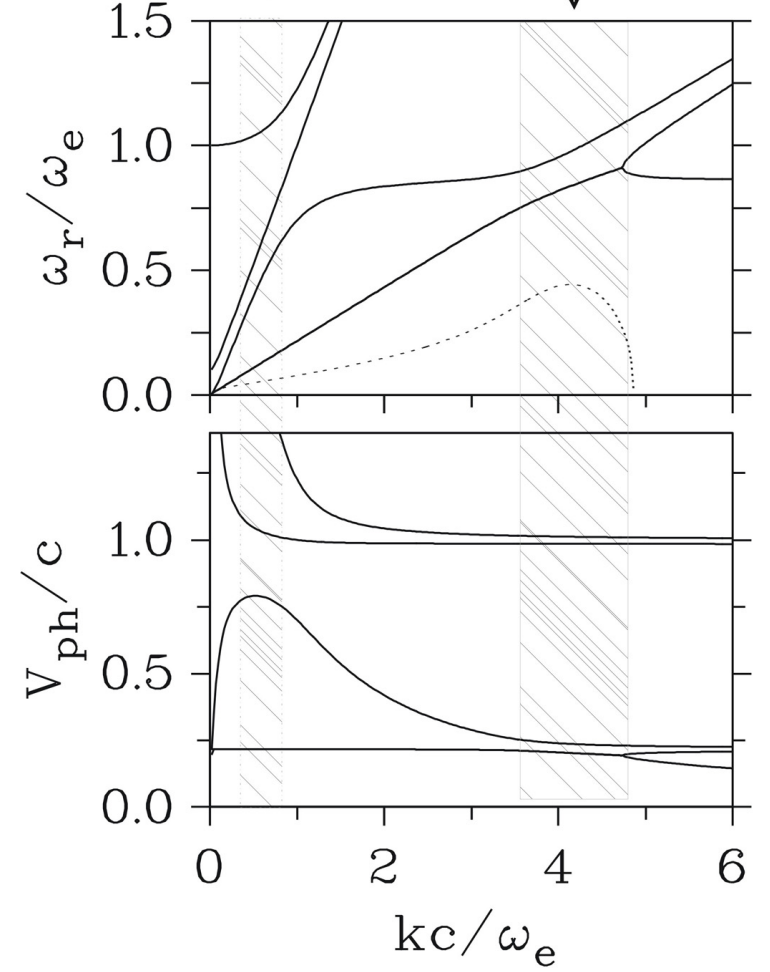

Fig. 15. From beam-plasma instability to oscillitons, different steps in the generation of plasma radiation under auroral conditions, $\Omega_{\mathrm{e}}>\omega_{\mathrm{e}}$ (schematic view). The beam-plasma instability generates predominately electrostatic waves in the wave number range of $k c / \omega_{\mathrm{e}} \sim c / V_{\mathrm{b}} \gg 1$, where $V_{\mathrm{b}}$ is the beam speed. After its saturation a nonlinear wave number shift to small values takes place. Finally, the wave energy accumulates around the point of maximum phase velocity (Gendrin point) which ends with the formation of whistler-Langmuir oscillitons at $k c / \omega_{\mathrm{e}} \leq 1$.

the large wave numbers at the plasma resonance $\left(\omega \sim \omega_{\mathrm{e}}\right)$. Our PIC simulations, however, give hints that the appearance of electromagnetic waves above the electron plasma frequency is possibly related to mode coupling which happens near the (cross-over) point where the Langmuir mode merges with the other two modes (whistler mode, L mode). This effect of mode coupling has already been discussed with respect to Fig. 11 and is shown once again in Fig. 13 in a more pronounced manner. As seen there, wave energy is transported from the source region around $k c / \omega_{\mathrm{e}} \sim c / V_{\mathrm{b}}$ to the cross-over region near to the Gendrin point $k c / \omega_{\mathrm{e}} \sim 1$,

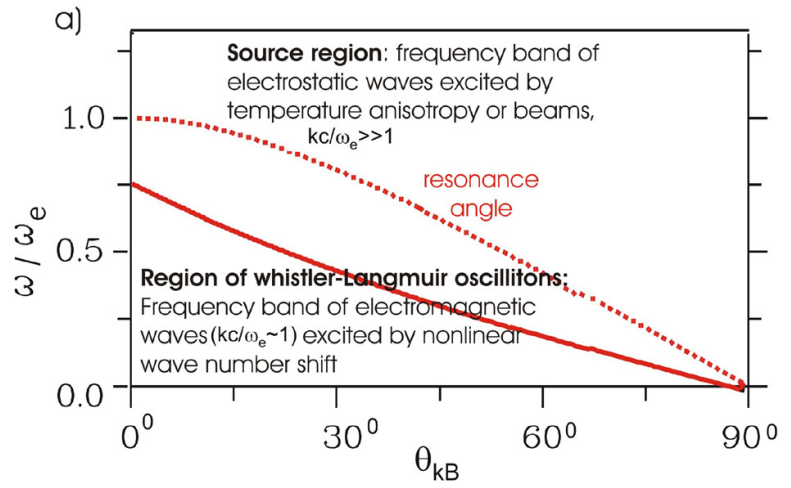

b)

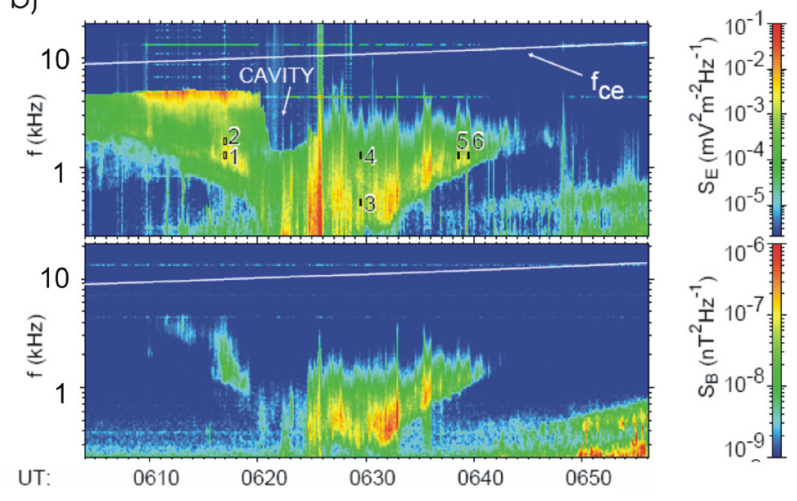

Fig. 16. Whistler-Langmuir waves in plasmas with $G>1$ : (a) schematic view of the two types of wave generation. According to experimental conditions in the bottom panel, $G=1.5$ was taken. In the source region predominantly electrostatic waves with $k c / \omega_{\mathrm{e}} \gg 1$ are generated by temperature anisotropy and/or electron beams. The frequency of these unstable waves is approximately given by the resonance cone condition (dotted red line). Nonlinear wave number shift into the region $k c / \omega_{\mathrm{e}} \sim 1$ drives whistlerLangmuir oscillitons which are probably the main contribution to electromagnetic wave emission. The solid red curve represents their frequency-angle dependence. (b) Spectrogram of the electric (upper panel) and magnetic components measured by the plasma wave instrument (PWI) onboard the Polar spacecraft (adapted from Santolik and Gurnett, 2002).

which is marked by the dashed red circle in the bottom panel. In the second panel of Fig. 13, which shows the magnitude of the magnetic field component $B_{\mathrm{y}}$ for $\omega$ versus $k$, there is a clear signature that indicates the wave modes above the plasma frequency $\omega_{\mathrm{e}}$, in particular the Z-mode, becomes excited. Our suggestion, supported by additional PIC simulations, is that the excitation is related to mode coupling that happens nearby the cross-over point. However, one has to take into consideration the weak damping of the involved wave modes in the wave number range of $k c / \omega_{\mathrm{e}} \sim 1$. Although such mode coupling is not directly evident from the dispersion curves in the lower panel of Fig. 13, where both wave modes, the whistler-Langmuir mode and the Z-mode appear as independent entities, their connection becomes apparent if the dispersion of the corresponding stationary waves 
a)

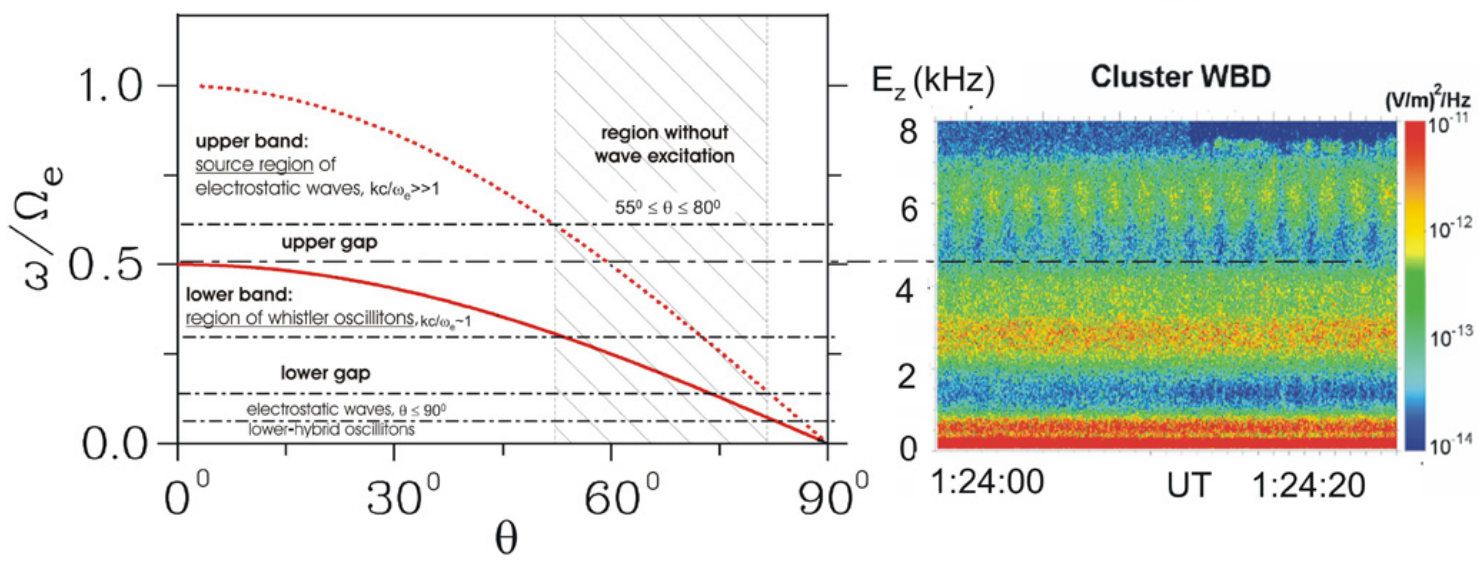

Fig. 17. Whistler wave bands and gaps in plasmas with $G=\Omega_{\mathrm{e}} / \omega_{\mathrm{e}}<1$. Left: the schematic view illustrates that the source region and the region of whistler-Langmuir oscillitons are well separated and that frequency bands and gaps exist below and above $\Omega_{\mathrm{e}} / 2$. The red dotted curve corresponds to the resonance cone formula $\omega / \Omega_{\mathrm{e}} \sim \cos \theta$ of the source region. The red solid curve represents the (Gendrin) oscilliton frequency versus the propagation angle $\theta$ according to $\omega / \Omega_{\mathrm{e}}=0.5 \cos \theta$ (see e.g. Sauer and Sydora, 2010). Right: frequency spectrogram, color coded in electric field intensity (adapted from Schriver et al., 2010). The WBD wave data show multiple bands and gaps of whistler wave emission, in reasonable agreement with the predictions in the left panel.

in Fig. 4 is considered. There is a region of spatially growing solutions which connects both of them and may explain why wave activity above $\omega_{\mathrm{e}}$ can also exist.

The fact that in the final stage of the beam-plasma interaction, as described in Fig. 13, at least two modes with different wave numbers but comparable amplitudes are simultaneously present is also well seen at the spatial profiles of the electric and magnetic field components. As an example, in Fig. 14 the magnetic field $B_{\mathrm{y}}(x)$ is shown at $\omega_{\mathrm{e}} t=400$ for the same numerical run as in Fig. 13. As expected, it reveals a pronounced wave packet structure. Besides the short-scale mode due to the beam excitation $\left(k V_{\mathrm{b}} \sim \omega_{\mathrm{e}}\right)$ with a wave length of $\lambda \sim 0.9 \mathrm{c} / \omega_{\mathrm{e}}$, clearly visible in the highresolution plot of one selected packet, the individual wave packets possess an average extension between about $8 c / \omega_{\mathrm{e}}$ and $12 c / \omega_{\mathrm{e}}$. This corresponds to a wave number range of $0.4 \leq k c / \omega_{\mathrm{e}} \leq 0.8$ which is in good agreement with that of a Gendrin mode that one gets from Fig. 3 if $\theta=30^{\circ}$ and $G=2$ are taken. Furthermore, it should be noted that the related frequency of the Gendrin mode wave is close to $\omega=0.5 \omega_{\mathrm{e}}$ and thus well separated from the Langmuir mode at $\omega \sim \omega_{\mathrm{e}}$.

Next, results of our theoretical work which is relevant to the generation of plasma radiation by electron beams under auroral conditions, i.e. for plasmas in which the electron cyclotron frequency is larger than the electron plasma frequency, should be summarized. Joint investigations in which dispersion analysis of beam-excited whistlerLangmuir waves and magnetized fluid-Maxwell approach of related oscillitons have been combined with PIC simulations, brought about an improved understanding of basic plasma phenomena arising during the temporal evolu- tion of the wave generation process. In Fig. 15 a schematic view is given: (A) the first step consists of the excitation of quasi-longitudinal waves by beam-plasma instability; maximum instability appears at wave numbers of $k c / \omega_{\mathrm{e}} \sim c / V_{\mathrm{b}}$. (B) The subsequent saturation process is accompanied by a wave number shift in the direction of smaller values. (C) If the (Gendrin) point of maximum phase velocity near $k c / \omega_{\mathrm{e}}=1$ is reached, the wave energy accumulates there and is associated with the formation of nonlinear stationary waves in the form of whistler-Langmuir oscillitons. These waves are weakly damped and may propagate over large distances. How the frequency of the two different wave types varies with the propagation angle $\theta$ is illustrated in Fig. 16 for $G=1.5$ (upper panel) together with spectral measurements (bottom two panels) of the electric and magnetic field components by the plasma wave instrument (PWI) onboard of the Polar spacecraft (Santolik and Gurnett, 2002). Whereas the dominant electric field has funnel-like shape and can be explained by resonance cone generation, it seems that the magnetic components originate from both contributions, from whistler-Langmuir oscillitons in the range $k c / \omega_{\mathrm{e}} \leq 1$, (see Fig. 3) and whistler mode waves propagating slightly off the resonance cone, $k c / \omega_{\mathrm{e}}>1$.

In this respect, it is interesting to show a similar representation as in Fig. 16, but for common whistlers in plasmas with $G<1\left(\Omega_{\mathrm{e}}<\omega_{\mathrm{e}}\right)$. Again, in the left panel of Fig. 17, the frequency versus propagation angle for the two wave types is plotted. The upper dotted red curve is the resonance cone condition $(\omega \sim \cos \theta)$, the solid red curve represents the (Gendrin) frequency, $\omega \sim 0.5 \cos \theta$, of whistler oscillitons (Sauer and Sydora, 2010). If one additionally assumes 
that in the interval of propagation angles $55^{\circ} \leq \theta \leq 80^{\circ}$ no wave excitation is possible due to the absence of conditions for wave-particle interaction, frequency bands of admissible emission and gaps in which no waves may occur can be deduced. The upper band (above $\Omega_{\mathrm{e}} / 2$ ) belongs to the source region of mainly electrostatic waves $\left(k c / \omega_{\mathrm{e}} \gg 1\right)$ and the adjacent (upper) gap is a consequence of missing excitation of waves propagating with angles $\theta \geq 55^{\circ}$, see also Schriver et al. (2010) and Liu et al. (2011). The lower frequency band (below $\Omega_{\mathrm{e}} / 2$ ), on the other hand, results from whistler oscillitons which are driven by (unstable) upper band waves via nonlinear spectral shift to the Gendrin point, $k c / \omega_{\mathrm{e}}=1$. The subsequent (lower) gap, however, is directly related to the upper gap assuming that the nonlinear wave number shift is primarily accomplished in one direction. Finally, two lowfrequency bands may appear due to weakly damped waves for nearly transverse propagation which are generated in both regions, the source region and the region of lower-hybrid oscillitons, respectively. If we compare the predicted frequency bands and gaps shown in the left panel of Fig. 17 with the wave data from Cluster measurements (right panel), there is a surprising accordance with our preceding predictions. Of course, more detailed studies are required to find out what is really behind this congruence.

Finally, we want to express our conviction that the knowledge gained from this specific wave generation process where whistler-Langmuir waves participate, can be generalized to wave phenomena in other frequency regimes. An important lesson we have learned is that the predictions of linear instability theory say very little about the wave characteristics under the conditions of nonlinear saturation and may even be misleading. Our main finding is that Gendrin modes and related oscillitons seem to play an essential role in characterizing the final nonlinear state of the system (Sydora et al., 2007; Dubinin et al., 2007; Stenzel et al., 2008; Verkhoglyadova and Tsurutani, 2009; Sauer and Sydora, 2010). In this respect many further applications (such as electron and ion cyclotron harmonic waves, multi-ion waves etc.) are seen. Considerably improved observational techniques on space satellites (e.g. by wave telescopes) which allow for better determination of the wave characteristics than in previous years, combined with the growing capabilities of kinetic simulations (PIC and Vlasov) make it possible to support our view.

Acknowledgements. This work was supported by a grant from the Natural Sciences and Engineering Research Council of Canada (NSERC). One of us (K.S.) thanks the DAAD (Germany) for travel support.

Topical Editor R. Nakamura thanks S. Ye and another anonymous referee for their help in evaluating this paper.

\section{References}

Baumjohann, W. and Treumann, R. A.: Basic Space Plasma Physics, Imperial College Press, 1996.

Cattaert, T. and Verheest, F.: Large amplitude parallel propagating electromagnetic oscillitons, Phys. Plasmas, 12, 012307, doi:10.1063/1.1824038, 2005.

Dubinin E., Sauer, K., and McKenzie, J. F.: Solitons and oscillitons in cold bi-ion plasmas: A parameter study, J. Plasma Phys., 68, 27-52, 2002.

Dubinin, E. M., Sauer, K., and McKenzie, J. F.: Nonlinear stationary waves and whistler solitons (oscillitons). Exact solutions, J. Plasma Phys., 69, 305-330, 2003.

Dubinin, E. M., Maksimovic, M., Cornilleau-Wehrlin, N., Fontaine, D., Travnicek, P., Mangeney, A., Alexandrova, O., Sauer, K., Fraenz, M., Dandouras, I., Lucek, E., Fazakerley, A., Balogh, A., and Andre, M.: Coherent whistler emissions in the magnetosphere - Cluster observations, Ann. Geophys., 25, 303-315, doi:10.5194/angeo-25-303-2007, 2007.

Gendrin, R.: Le guidage des whistlers par le champ magnetique, Planet. Space Sci., 5, 274-281, 1961.

Gurnett, D. A.: A satellite study of VLF hiss, J. Geophys. Res., 71, 5599-5615, 1966.

Gurnett, D. A., Shawhan, S., and Shaw, R.: Auroral hiss, Z-mode radiation, and auroral kilometric radiation in the polar magnetosphere, DE 1 observations, J. Geophys. Res., 88, 329-340, 1983.

Kennel, C.: Low-frequency whistler mode, Phys. Fluids, 9, 21902202, doi:10.1063/1.761588, 1966.

Kopf, A. J., Gurnett, D. A., Menietti, J. D., Schippers, P., Arridge, C. S., Hospordarsky, G. B., Kurth, W. S., Grimald, S., Andre, N., Coates, A. J., and Dougherty, M. K.: Electron beams as source of whistler-mode auroral hiss at Saturn, Geophys. Res. Lett., 37, L09102, doi:10.1029/2010GL042980, 2010.

Lin, C. S., Burch, J. L., Shawhan, S. D., and Gurnett, D. A.: Correlation of auroral hiss and upward electron beams near the polar cusp, J. Geophys. Res., 89, 925-935, 1984.

Liu, K., Gary, S. P., and Winske, D.: Excitation of banded whistler waves in the magnetosphere, Geophys. Res. Lett., 38, L14108, doi:10.1029/2011GL048375, 2011.

Ma, J. Z. G. and Hirose, A.: Lower-hybrid (LH) oscillitons evolved from ion-acoustic (IA)/ion-cyclotron (IC) solitary waves: effect of electron inertia, Nonlin. Processes Geophys., 17, 245-268, doi:10.5194/npg-17-245-2010, 2010.

Ma, J. Z. G., Hirose, A., St.-Maurice, J.-P., and Liu, W.: Nonlinear electrostatic ion-acoustic "oscilliton" waves driven by charge non-neutrality effects, Ann. Geophys., 29, 123-145, doi:10.5194/angeo-29-123-2011, 2011.

Maggs, J. E.: Coherent Generation of VLF Hiss, J. Geophys. Res., 81, 1707-1724, 1976.

McKenzie, J. F., Dubinin, E., Sauer, K., and Doyle, T. B.: The application of the constants of motion to nonlinear stationary waves in complex plasmas: a unified fluid dynamic viewpoint, J. Plasma Phys., 70, 431-462, 2004.

McKenzie, J. F., Dubinin, E. M., and Sauer, K.: Relativistic whistler oscillitons - do they exist?, Nonlin. Processes Geophys., 12, 425-431, doi:10.5194/npg-12-425-2005, 2005.

Morgan, D. D., Gurnett, D. A., Menietti, J. D., Winningham, J. D., and Burch, J. L.: Landau damping of auroral hiss, J. Geophys. Res., 99, 2471-2488, 1994.

Santolik, O. and Gurnett, D. A.: Propagation of auro- 
ral hiss at high altitudes; Geophys. Res. Lett., 29, 119, doi:10.1029/2001GL013666, 2002.

Sauer, K. and Sydora, R. D.: Beam-excited whistler waves at oblique propagation with relation to STEREO radiation belt observations, Ann. Geophys., 28, 1317-1325, doi:10.5194/angeo28-1317-2010, 2010.

Sauer, K., Dubinin, E., and McKenzie, J. F.: New type of soliton in bi-ion plasmas and possible implications, Geophys. Res. Lett., 28, 3589-3592, 2001.

Sauer, K., Dubinin, E. M., and McKenzie, J. F.: Wave emission by whistler oscillitons: Application to "coherent lion roars", Geophys. Res. Lett., 29, 2226, doi:10.1029/2002GL015771, 2002.

Sauer, K., Dubinin, E., and McKenzie, J. F.: Solitons and oscillitons in multi-ion space plasmas, Nonlin. Processes Geophys., 10, 121-130, doi:10.5194/npg-10-121-2003, 2003.

Schriver, D., Ashour-Abdalla, M., Coroniti, F. V., LeBoeuf, J. N., Decyk, V., Travnicek, P., Santolik, O., Winningham, D., Pickett, J. S., Goldstein, M. L., and Fazakerly, A. N.: Generation of whistler mode emissions in the inner magnetosphere: An event study, J. Geophys. Res., 115, A00F17, doi:10.1029/2009JA014932, 2010.
Silin, I., Sydora, R. D., Mann, I. R., Sauer, K., and Mace, R. L.: Nonlinear evolution of electromagnetic ion cyclotron (EMIC) waves, Phys. Plasmas, 18, 042108-042108-8, 2011.

Stenzel, R. I., Urrutia, J. M., and Strohmaier, K. D.: Whistler spheromaks, instabilities and triggered emissions experiments, Plasma Phys. Control. Fusion, 50, 074009, doi:10.1088/07413335/50/7/074009, 2008.

Stix, T. H.: Waves in Plasmas. American Institute of Physics, New York, 1992.

Sydora, R. D., Sauer, K., and Silin, I.: Coherent whistler waves and oscilliton formation: Kinetic simulations, Geophys. Res. Lett., 34, L22105, doi:10.1029/2007GL031839, 2007.

Usanova, M. E.: Generation of Electromagnetic Ion Cyclotron (EMIC) Waves in a Compressed Dayside Magnetosphere, $\mathrm{PhD}$ Thesis, University of Alberta, Canada, 2010.

Verkhoglyadova, O. P. and Tsurutani, B. T.: Polarization properties of Gendrin mode waves observed in the Earth's magnetosphere: observations and theory, Ann. Geophys., 27, 4429-4433, doi:10.5194/angeo-27-4429-2009, 2009.

Willes, A. J. and Cairns, I. H.: Generalized Langmuir waves in magnetized kinetic plasmas, Phys. Plasmas, 7, 3167-3180, 2000. 